\title{
Von Südtirol nach Württemberg. Die «Umsiedlung» Südtiroler Psychiatriepatienten im Rahmen des deutsch-italienischen Optionsvertrages ab 1939*
}

Maria Fiebrandt, Bodo Rüdenburg und Thomas Müller

\section{Summary}

Object of this article is the procedure of the transport of South Tyrol patients to the South Wuerttemberg asylums Zwiefalten und Schussenried as well as to some extent to the former asylum of Weissenau near Ravensburg in 1940 and 1942. Attention is focused on the pioneering state pre-negotiations, the so-called option treaties between the German Reich and Fascist Italy as part of the general aspect of National Socialist bio-Politics. The treatment of these South Tyrol patients in the asylums themselves, as well as their fate will be put into the context of the resettlement actions at the margins of the "Third Reich", which started in 1939 and widely affected the European continent. By presenting the abuse of medical patients in the aim to solve the political problems having occurred after bio-political goals had been set into practice, this study offers new material to the history of National Socialist psychiatry, as well as to National Socialist Foreign Policy in the case of Italy.

Keywords: resettlement, psychiatry, South Tyrol, euthanasia, "Third Reich"

* Wir danken dem Herausgeber Vincent Barras sowie den anonymen Gutachtern von Gesnerus für wichtige Hinweise und Überarbeitungsvorschläge.

Maria Fiebrandt, M.A., Lehrstuhl für Zeitgeschichte, Technische Universität Dresden (MariaFiebrandt@gmx.de); Bodo Rüdenburg und Dr. med. Thomas Müller, M.A., Forschungsbereich Geschichte und Ethik in der Medizin; Klinik für Psychiatrie und Psychotherapie I der Universität Ulm/Zentrum für Psychiatrie (th.mueller@zfp-zentrum.de). 


\section{Zusammenfassung}

Gegenstand dieses Beitrages ist die «Umsiedlung» von Südtiroler Patientinnen und Patienten in die südwürttembergischen Heil- und Pflegeanstalten Zwiefalten, Schussenried und Ravensburg-Weissenau im Rahmen des sogenannten Optionsvertrages zwischen dem Deutschen Reich und Italien. Anhand dieses konkreten Beispiels soll gezeigt werden, wie und in welchem Ausmaß im Kontext der NS-Umsiedlungssiedlungspolitik siedlungs-, bevölkerungs- und «erbgesundheitspolitische» Zielsetzungen und Praktiken miteinander verschmolzen und welche konkreten Konsequenzen im Einzelfall daraus erwachsen konnten. Im Speziellen wird dazu die «Umsiedlung» der psychisch und physisch kranken Südtiroler vor dem Hintergrund der ab 1939 einsetzenden, ganz Europa umfassenden, Umsiedlungsaktionen des «Dritten Reiches» in den Blick genommen - angefangen bei der Erfassung der Patienten in den Heimatorten, über den Abtransport in separaten Krankentransporten bis hin zur Unterbringung und Behandlung in den «reichsdeutschen» Anstalten. Dabei geht es nicht allein um die bereits psychiatrisierten Anstaltspatienten, sondern auch um kranke, «auffällige» Südtiroler, die erst im Kontext der Umsiedlung psychiatrisiert wurden, und damit ebenso in das Räderwerk der NS-«Erbgesundheitspolitik» gerieten. Ihr nicht nur aus medizinhistorischer sondern auch aus zeitgeschichtlicher Perspektive außerordentlich aufschlussreiches Schicksal soll hier nachgezeichnet werden, und zwar auch über das Jahr 1945 hinaus.

\section{Einführung}

Im Fokus des vorliegenden Beitrages steht die Verschmelzung siedlungs-, bevölkerungs- und «erbgesundheitspolitischer» Zielsetzungen und Praktiken im Kontext der NS-Umsiedlungspolitik, die am Beispiel der Umsiedlung der Südtiroler, insbesondere dem Schicksal psychisch kranker oder auch nur in irgendeiner Weise «auffälliger» Südtiroler, untersucht wird. Dabei werden die verschiedenen Umsiedlungsetappen - angefangen bei der Erfassung der Betroffenen in Südtirol, über deren «Umsiedlung» in das Deutsche Reich bis hin zur Unterbringung in den dortigen Heilanstalten Schussenried, Zwiefalten und Ravensburg-Weissenau - in den Blick genommen und die dabei wirkenden Erfassungs- und Selektionsmechanismen einer genaueren Betrachtung unterzogen. So wird insbesondere auf die in der neuesten Literatur doch zumindest am Rande erwähnte verstärkte Psychiatrisierung und Hospitalisierung alter und kranker Südtiroler im Kontext der Umsiedlungs- 
aktion eingegangen, ${ }^{1}$ Vorbedingungen dieser beleuchtet und das durchaus breite Handlungsfeld der Akteure skizziert werden. Welche Folgen hatte schließlich diese Psychiatrisierung? Wurden die Südtiroler in den Anstalten des Deutschen Reiches Opfer der NS-«Euthanasie» oder genossen sie einen besonderen Schutzstatus - eine Frage, die innerhalb der medizinhistorischen und zeitgeschichtlichen Forschung lange Zeit durchaus widersprüchlich beantwortet wurde ${ }^{2}$ und angesichts des aktuell gesteigerten Interesses am Schicksal der Südtiroler Psychiatriepatienten zusätzlich an Bedeutung gewinnt. $^{3}$

«Heim ins Reich!» - diese von Konrad Henlein geprägte propagandistische Parole wurde zum Sinnbild der inszenierten «Heimkehr» der in verschiedenen deutschen Sprachinseln im Ausland lebenden sog. Volksdeutschen ${ }^{4}$ in das Deutsche Reich. Verschiedene bilaterale Verträge regelten die Umsiedlungsmodalitäten und legten den Kreis der Umzusiedelnden fest. ${ }^{5}$ Diese sollten allerdings keineswegs wie suggeriert im ursprünglichen Reichsgebiet angesiedelt werden, ihnen wurde vielmehr eine wesentliche Aufgabe im Rahmen der nationalsozialistischen Germanisierungspläne zugeschrieben. ${ }^{6}$ Diejenigen Volksdeutschen, die aus Sicht der Umsiedlungsakteure über die erwünschten rassischen und biologischen Merkmale verfügten, sollten eine Bauernstelle in den nach rassenbiologischen Ordnungsprinzipien neu zu gestaltenden Gebieten - zum Beispiel der sog. Untersteiermark oder dem Warthegau - erhalten, «weil dort die Grundlage zu einem festen Wall deutscher Bauernfamilien geschaffen werden [sollte] und das beste Blut hier gerade gut genug» erschien. ${ }^{7}$ Diese rassenbiologische Zielsetzung sollte die Umsiedlungen von Beginn an maßgeblich prägen. Sie setzte einen Selektionsprozess in Gang, innerhalb dessen eine genaue Überprüfung eines

1 Vgl. Wedekind 2012.

2 Zu den Südtiroler Psychiatriepatienten vgl. u.a. Pantozzi 1989, Hinterhuber 1995, May 1996b, zu volksdeutschen, osteuropäischen Psychiatriepatienten vgl. Aly 1998 und Rieß 1995.

3 Verwiesen sei hier vor allem auf die laufenden Forschungen der Universität Innsbruck, des Südtiroler Landesarchivs und des Psychiatrischen Krankenhauses Hall i.T. Erste Ergebnisse in Dietrich-Daum u.a. 2011.

4 Es sei darauf hingewiesen, dass es sich bei den Begrifflichkeiten «Volksdeutsche», «Umsiedler» und «Rückwanderer» die hier synonym verwendet werden, um typische Beispiele der lingua tertii imperii handelt, die ideologisch eingefärbt und ungenau sind. Sie werden lediglich der besseren Lesbarkeit wegen als Überbegriffe für die aus verschiedensten Teilen Südostund Osteuropas sowie Südtirol stammenden Staatsangehörigen mit deutschen Vorfahren verwendet.

5 Einen Überblick über die Umsiedlungspolitik des Nationalsozialismus bietet Leniger 2006. Vgl. auch Kotzian 2005 sowie zur nationalsozialistischen «Raumforschung〉 generell Gutberger $2009,21-45$.

6 Vgl. weiterführend z.B. Heinemann/Wagner 2006; zum Zusammenhang zwischen Umsiedlung und Holocaust vgl. Aly 1998.

7 Reichskommissar für die Festigung Deutschen Volkstums 1940, VII. 
jeden Umsiedlers stattfand, die darauf zielte, «unerwünschten Bevölkerungszuwachs» «auszusondern». Im Sinne der NS-Siedlungspolitik, die auf rassenbiologische und rassenhygienische Denkmuster rekurrierte, stellten vor allem soziale Randgruppen, wie psychisch kranke Menschen, diesen «unerwünschten Bevölkerungszuwachs» dar. Dies führte jedoch keineswegs zu einem zwangsläufigen Ausschluss aus der Umsiedlungsaktion, weder bei der Umsiedlung der Südtiroler noch bei anderen geschlossenen Umsiedlungen, beispielsweise aus dem Baltikum, Galizien oder Bessarabien. ${ }^{8}$ Allerdings lassen sich in den Umsiedlungsverträgen und -bestimmungen nur selten konkrete Regelungen bezüglich der Behandlung psychisch Kranker finden. ${ }^{9}$ Lediglich die Transportfrage war in der Regel recht präzise geklärt: der Abtransport aller kranken, alten, gebrechlichen und schwangeren Umsiedler/Umsiedlerinnen sollte in separaten Krankentransporten erfolgen. Doch welches Ziel hatten diese Krankentransporte, welches Schicksal erwartete die Umsiedler?

Diese Frage drängt sich angesichts der im Deutschen Reich bis 1939/40 etablierten repressiven gesundheitspolitischen Maßnahmen und der zu diesem Zeitpunkt beginnenden Krankenmordaktionen geradezu auf. Zwangssterilisationen und Abtreibungen aus eugenischen Indikationen waren bereits tausendfach vorgenommen worden, 1939 setzte die Tötung «mißgebildeter» Kinder in speziellen «Kinderfachabteilungen» ein, die Planungen für die «Aktion T4» liefen auf Hochtouren. Insbesondere die «Aktion T4», in deren Aktionsradius sich unweigerlich alle Anstaltspatienten im Deutschen Reich befanden, sollte für die Südtiroler mit ihrer Umsiedlung zu einer existentiellen Bedrohung werden.

Vor diesem Hintergrund seien hier einige Anmerkungen zur «Aktion T4» gestattet. ${ }^{10}$ Im Oktober 1939 ordnete Hitler den «Gnadentod» für «psychisch Kranke» und «geistig behinderte» Anstaltspatienten an. In einem auf privatem Briefpapier verfassten Schreiben ermächtigte er den Leiter der Kanzlei des Führers, Philipp Bouhler, und seinen Begleitarzt Karl Brandt

8 So wurden beispielsweise psychisch kranke Baltendeutsche aus Riga über Swinemünde in die psychiatrischen Anstalten in Meseritz-Obrawalde und Arnsdorf b. Dresden gebracht. Vgl. Schreiben der NSDAP Kreisleitung Swinemünde an die Volksdeutsche Mittelstelle Posen betr. Krankentransporte ins Reich vom 23.2.1940 sowie «Bericht über den Geisteskrankentransport Riga-Arnsdorf vom 18.12.1939», Archivum Państwowe w Poznaniu (APP), Bestand 800 (Vomi), 123, B1. 43 und 112-118. Die Baltendeutschen wurden wenige Monate später in den sog. Warthegau verlegt, dessen Anstalten (Tiegenhof, Warta, Gostynin) in der Folgezeit auch das Ziel weiterer Krankentransporte aus Bessarabien, der Bukowina und Litauen wurden. Vgl. Fiebrandt 2012.

9 Hecker 1971, 15-22.

10 Einen kompakten Überblick zum derzeitigen Forschungsstand bietet Jütte 2011. Weiterführend vgl. auch Rotzoll u.a. 2010. 
«die Befugnisse namentlich zu bestimmender Ärzte so zu erweitern, dass nach menschlichem Ermessen unheilbar Kranken bei kritischster Beurteilung ihres Krankheitszustands» der sogenannte Gnadentod «gewährt» werden könne. An die psychiatrischen Einrichtungen, Krankenhäuser und anderen Institutionen zur Versorgung chronisch kranker Menschen wurden nachfolgend Meldebögen versandt. Gemeldet werden sollten alle die Patienten, die bspw. an Schizophrenie, Epilepsie, senilen Störungen, therapieresistenter Paralyse, Retardierung, Enzephalitis oder Huntington'scher Chorea litten. Auch alle diejenigen, die für mindestens fünf Jahre kontinuierlich in einer der oben genannten Institutionen aufgenommen gewesen waren, mussten gemeldet werden, genauso wie diejenigen, die als Kriminelle galten, oder nicht die deutsche Staatsbürgerschaft besaßen, oder nicht «von deutschem Blut» waren, besonders wenn es sich bei diesen Menschen um Juden, Sinti, Roma oder Menschen schwarzer Hautfarbe handelte. Organisiert wurde der Massenmord in Berlin, in der Tiergartenstraße 4, woraus der auch in der medizin- und zeithistorischen Literatur Verwendung findende Terminus «T4» resultiert. ${ }^{11}$

Insgesamt dienten ab Anfang 1940 sechs Einrichtungen der gezielten Ermordung der Patienten: Brandenburg, ${ }^{12}$ Grafeneck, Hartheim b. Linz, Pirna-Sonnenstein, Bernburg und Hadamar. In das Einzugsgebiet zweier dieser «T4»-Tötungsanstalten gelangten auch die Südtiroler: Grafeneck und Hartheim. ${ }^{13}$ Das Prozedere war dabei in allen diesen Tötungsanstalten ähnlich. Die gemeldeten Patienten bzw. deren Meldebögen wurden zunächst von Ärzten «begutachtet». Nachdem diese die Entscheidung über «lebenswert» bzw. «lebensunwert» getroffen hatten, erstellte die «T4» Transportlisten und leitete die «Verlegung» der zu tötenden Patienten aus den Krankenhaus-Einrichtungen in die genannten Mordstätten in die Wege. In deren Gaskammern wurden die Patienten ermordet und anschließend in den dazugehörigen Krematorien verbrannt. Nach der Ermordung wurden die Angehörigen beziehungsweise Familien der Ermordeten über die angebliche Todesursache unterrichtet, bei der es sich regelmäßig um eine zunächst plausibel erscheinende, jedoch frei erfundene, also nicht zutreffende Diagnose handelte. Am 24. August 1941 ordnete Hitler u.a. aufgrund von

11 Müller/Beddies 2004 sowie Müller/Beddies (2006). Vgl. auch Rotzoll u.a. 2010.

12 In Brandenburg beispielsweise wurde ein ehemaliges Zuchthaus in eine solche Einrichtung umgewandelt. Hier wurden auch Experimente mit Gas durchgeführt. Erste Vergasungsversuche an Psychiatriepatienten hatten allerdings bereits im Oktober/November $1939 \mathrm{im}$ Fort VII in Posen/Warthegau unabhängig von der «T4» stattgefunden. Den nach wie vor besten Überblick dazu bietet Rieß 1995. Vgl. auch Longerich 1998. Zur Probevergasung in Brandenburg vgl. Ley 2010.

13 Zu Grafeneck vgl. u.a.: Müller 2001, zu Hartheim vgl. Kepplinger/Marckhgott/Reese 22008. 
Protesten einzelner Mitglieder der Kirchen und einer veränderten Kriegssituation den Abbruch der «Aktion T4» an. Zu diesem Zeitpunkt waren allein durch diese Tötungsaktion, die sogenannte zentrale «Euthanasie», ungefähr 70000 Menschen ermordet worden. Die nationalsozialistischen Krankenmorde fanden mit dem Stopp der «Aktion T4» jedoch keineswegs ihr Ende, sondern wurden auf andere Art und Weise weitergeführt. Nun wurden Medikamente und der Hungertod zur hauptsächlichen Bedrohung der zu diesem Zeitpunkt noch lebenden Patientinnen und Patienten. Bis 1945 wurden im Rahmen der sogenannten dezentralen «Euthanasie» fast 300000 hilflose Menschen ermordet. ${ }^{14}$ Vor dem Hintergrund dieser Entwicklung ist die Frage offensichtlich gerechtfertigt, ob und inwieweit auch die psychisch kranken Umsiedler Opfer dieser Krankenmorde wurden. Im Fokus dieses Beitrags sollen dabei die Südtiroler «Geisteskranken», stehen, welche, der bisherigen Literatur zufolge, von der NS-«Euthanasie» verschont geblieben sein sollen - offensichtlich weil sie eine Art Sonderstatus genossen. ${ }^{15}$ Diesen Sonderstatus gilt es genauer in den Blick zu nehmen. ${ }^{16}$ Es sind, ausführlicher als bislang in der Forschung geschehen, die Motivlage und die Zielsetzung, die die Umsiedlung der Südtiroler Geisteskranken bestimmten, zu beleuchten. ${ }^{17} \mathrm{Gab}$ es zentrale Grundsatzentscheidungen, beispielsweise in Bezug auf die Einbürgerung und Unterbringung der «Geisteskranken», oder trafen die Umsiedlungsakteure und Gesundheitspolitiker hier eher improvisatorisch-pragmatische ad-hoc-Entscheidungen?

14 Fuchs u.a. 2007, 15.

$15 \mathrm{Zu}$ den Südtirolern vgl. vor allem May 1996a, Pantozzi 1989. Zu anderen Umsiedlergruppen vgl. Aly 1998.

16 Die Einbeziehung aller sog. volksdeutschen Umsiedler, also auch jener aus dem Osten und Südosten Europas, in die NS-Erbgesundheitspolitik war Gegenstand eines übergeordneten DFG-Forschungsprojektes der TU Dresden (Maria Fiebrandt). Bei den nachfolgend dargestellten Zusammenhängen zur Umsiedlung der Südtiroler handelt es sich um erste Teilergebnisse dieses Projektes. Die relevanten Aspekte zur Geschichte der ehemaligen Anstalten Zwiefalten, Schussenried und Weissenau resultieren aus der Forschung von Bodo Rüdenburg und Thomas Müller am Forschungsbereich Geschichte der Medizin der Nachfolgeeinrichtung dieser südwürttembergischen Einrichtungen.

17 In Ansätzen bereits bei Stuhlpfarrer 1985, dem nach wie vor grundlegenden Werk zur Umsiedlung der Südtiroler. 


\section{Der Ausgangspunkt der Umsiedlung: Das deutsch-italienische Abkommen über die Umsiedlung der Südtiroler}

Im Mai 1939 legte Heinrich Himmler ein Memorandum über die «endgültige» Lösung der Südtirolfrage vor, ${ }^{18}$ in welchem er im Sinne Adolf Hitlers den vollständigen Verzicht auf Südtirol propagierte und zugleich die Umsiedlung aller in Südtirol lebenden «Volksdeutschen» forderte. ${ }^{19}$ Nur einen Monat später, am 23. Juni 1939, erhielt dieses Memorandum offiziellen Charakter in Form der «Berliner Vereinbarung», in der das Ziel, eine «radikale ethnische Lösung der Frage des Oberetsch $»^{20}$ herbeizuführen, deklariert wurde. Der Vereinbarung nach sollten alle in Südtirol lebenden Reichsdeutschen ins Deutsche Reich zurückkehren. Den Volksdeutschen, die die italienische Staatsbürgerschaft besaßen, wurde ein Optionsrecht eingeräumt. Sie sollten sich für die italienische oder die deutsche Staatsbürgerschaft entscheiden. Nicht selten wurde diese Entscheidung, die formal eine freie und unbeeinflusste sein sollte, seitens der deutschen Dienststellen und des Völkischen Kampfrings Südtirol (VKS) zur Gewissensfrage «für oder gegen Deutschland» stilisiert. ${ }^{21}$

Noch vor der Veröffentlichung entsprechender Umsiedlungsrichtlinien wurde im September 1940 die Amtliche Deutsche Ein- und Rückwandererstelle (ADERSt) in Bozen errichtet, die mit Unterstützung der Arbeitsgemeinschaft der Optanten für Deutschland (AdO) die Umsiedlung vor Ort organisieren sollte. Ihr übergeordnet entstand die Dienststelle des Reichskommissars für die Festigung deutschen Volkstums (RKF), als welcher Heinrich Himmler seit dem 7. Oktober 1939 firmierte. Sie sollte sämtliche Umsiedlungsaktionen planen, organisieren und koordinieren. Die Einbürgerung und die Ansiedlung der Südtiroler sollten von der Dienststelle Umsiedlung Südtirol (DUS) in Innsbruck, die dem Reichsstatthalter in Tirol und Vorarlberg unterstand, übernommen werden..$^{22}$

18 Es handelt sich um eine dreiseitige Ausarbeitung Himmlers, in der die «endgültige» Lösung der Südtirolfrage skizziert wurde. Das Memorandum ist abgedruckt bei Latour 1962, $34 f$.

19 Die Bedeutung des Memorandums Himmlers reicht über die Südtirolproblematik noch hinaus, da hier bereits die Grundlinien der zukünftigen RKF-Politik (Siedlungspolitik) dargelegt werden. Deshalb schreibt Leniger diesem auch Modellcharakter zu, vgl. Leniger 2006, S. 44-48. Zur Genese des Umsiedlungsgedankens und ersten Vorverhandlungen vgl. Stuhlpfarrer 1985, 30-48. Zur Rolle des Völkischen Kampfrings Südtirol (ab 1940 Arbeitsgemeinschaft der Optanten für Deutschland) siehe Wedekind 2009, 71-109, zu den psychisch Kranken 79-80.

20 Handausgabe der Umsiedlungs-Bestimmungen für die deutschen Optanten 1940, 3.

21 Stuhlpfarrer 1985, 201. Tatsächlich fiel das Optionsergebnis deutliche zugunsten Deutschlands aus. Die Gründe dafür sind vielfältig, nicht zuletzt spielte auch die Propaganda eine wesentliche Rolle, vgl. Stuhlpfarrer 1985, 177-205.

$22 \mathrm{Zu}$ weiteren involvierten Dienststellen und ausführlichen Angaben vgl. Stuhlpfarrer 1985, Kap. Umsiedlungsorganisation, sowie Alexander 1993. 
Entsprechende Richtlinien, die die Details der Option und der Umsiedlung regelten, wurden am 21. Oktober 1939 erlassen. Optionsberechtigt sollten demnach alle «Volksdeutschen» sein, die in den Provinzen Bolzano, Trento, Belluno und Udine ihren Wohnsitz hatten. Ausschlaggebend sollte einzig und allein sein, dass der Optant beziehungsweise die Optantin sich zum deutschen Volk «zugehörig» betrachten würde. Weitere Präzisierungen des Begriffes «volksdeutsch» wurden nicht vorgenommen. Die umsiedlungswilligen Südtiroler waren aufgefordert bis zum 31. Dezember 1939 eine Optionserklärung abzugeben und anschließend einen Abwanderungsantrag bei der ADERSt in Bozen beziehungsweise einer ihrer Zweigstellen einzureichen. Inhaftierte Volksdeutsche sollten zu diesem Zweck in ihre Heimatgemeinde entlassen werden, und im Falle einer Option für Deutschland die Strafverfolgung ausgesetzt werden. Für minderjährige Volksdeutsche war die Option der Eltern maßgeblich. Inwieweit die Option auch für nicht geschäftsfähige Familienmitglieder und Verwandte, insbesondere für Insassen von Südtiroler Heil- und Pflegeanstalten gelten sollte, blieb in den Richtlinien offen. Es findet sich lediglich ein «alte und kranke Reichsdeutsche volksdeutsch» betreffender Passus, der diesen den Verbleib in Südtirol gestattete. Allerdings hatte Himmler im Zuge der Vorverhandlungen bereits signalisiert, dass Deutschland bereit sei, «die geistig und körperlich minderwertigen» Volksdeutschen und Gefängnisinsassen zumindest aufzunehmen. ${ }^{23}$ Alles in allem sollte die Abwanderung bis zum 31. Dezember 1942 abgeschlossen sein. ${ }^{24}$

Die Option erbrachte ein deutliches Votum für das Deutsche Reich. In den Provinzen Bozen und Udine hatten sich über 80 Prozent der optionsberechtigten Südtiroler für die Umsiedlung in das Deutsche Reich entschieden, in allen Provinzen insgesamt über 200000 Menschen. Diese sollten Südtirol zeitlich gestaffelt verlassen: zunächst die Reichsdeutschen, anschließend die «nicht bodengebundene» und zum Schluss die «bodengebundene» Bevölkerung. Oberste Prämisse und zugleich Grundproblem war die geschlossene Ansiedlung der Südtiroler. Denn auch wenn diverse Ansiedlungspläne «freie» Siedlungsgebiete offerierten, standen diese de facto nicht zur Verfügung. ${ }^{25}$ Die deutsche Seite war demnach bemüht, die endgültige Umsiedlung der «bodengebundenen» Südtiroler zunächst hinauszuschieben, bis sich

23 Stuhlpfarrer 1985, 143. Vgl. auch Verhandlungen über die Einbürgerung dieser Personenkreise in Bundesarchiv (BA) Berlin, R 49/1173.

24 Richtlinien für die Rückwanderung der Reichsdeutschen und Umsiedlung der Volksdeutschen aus dem Alto Adige vom 21.10.1939 sowie Erläuterungen zu den Richtlinien für die Rück- und Auswanderung nach Deutschland vom 17.11.1939, in: Handausgabe der Umsiedlungs-Bestimmungen für die deutschen Optanten 1940, 6-12,13f.

$25 \mathrm{Zu}$ den Siedlungsplanungen vgl. Wedekind 2012, vgl. auch Leidlmair 1991. 
geeignete Ansiedlungsgebiete eröffnen würden. Da das Deutsche Reich sich jedoch verpflichtet hatte, eine Abwanderungsquote von täglich 200 Personen einzuhalten, wurden vorerst die «nicht bodengebundenen» Südtiroler, die beispielsweise in der deutschen Wirtschaft oder der Wehrmacht eingesetzt werden konnten, umgesiedelt. ${ }^{26}$ Gleichzeitig hatte die italienische Seite wiederum ein Interesse daran, Personen, die politisch oder finanziell eine Belastung für den italienischen Staat darstellten, zur Abwanderung zu bewegen. So erklärt sich u.a. auch der Vorstoß des Bozener Präfekten Agostino Podestá vom 16. April 1940, Südtiroler Geisteskranke und Altersheimbewohner ins Deutsche Reich zu verbringen. ${ }^{27}$ Angesichts der zunehmenden Schwierigkeiten, die Abwanderungsquoten zu erfüllen, kam dieser Vorschlag den deutschen Umsiedlungsdienststellen durchaus entgegen und wurde schließlich noch auf Prostituierte ausgeweitet. ${ }^{28}$

Die Umsiedlung der Südtiroler psychisch Kranken erfolgte dabei (1) in Form eines Direkttransportes aus der Südtiroler Heilanstalt Pergine in die württembergische Heilanstalt Zwiefalten im Mai 1940 und (2) in Form von mehreren kleineren Transporten in den Jahren 1940 bis 1942 über die Tiroler Heilanstalt Hall, welche im Zuge der Umsiedlung zu einer Art Sammelstation für Südtiroler psychisch Kranke wurde, ins württembergische Schussenried (heute: Bad Schussenried).

\section{Die Verlegung Südtiroler Psychiatriepatienten aus Pergine nach Zwiefalten}

Die Verlegung von 299 Patienten aus Pergine nach Zwiefalten lässt sich anhand der überlieferten Quellen relativ präzise rekonstruieren und ist im Gegensatz zu Transporten psychisch kranker Volksdeutscher aus anderen Umsiedlungsgebieten auch bereits in Ansätzen erforscht. ${ }^{29}$

Ausgangspunkt zu konkreten Verhandlungen über die Verlegung von Südtiroler Anstaltspatienten in das Deutsche Reich war vermutlich eine Besprechung am 16. April 1940 in Bozen, anlässlich derer der Bozener Präfekt Podestá dem Leiter des Stabshauptamtes des RKF, Ulrich Greifelt, den schon erwähnten Vorschlag der Verlegung von vierzig Geisteskranken

26 Vgl. Alexander 1993.

27 Vgl. Protokoll der Besprechung im Greifen (Bozen) am 16.4.1940, undat., BA Berlin, R 49/2114, unpag.

28 Vgl. Stuhlpfarrer 1985, 518f., sowie Schriftwechsel zwischen der Dienststelle Umsiedlung Südtirol (DUS) Innsbruck und RKF betr. Prostituierter, Tiroler Landesarchiv Innsbruck (TLA), Reichsstatthalter, DUS, 50.

29 Vgl. Verband Angehöriger und Freunde psychisch Kranker 1995; Pantozzi 1989, 1996; Hinterhuber 1995; May 1996a; May 1996b sowie als Ego-Dokument Demetz, 1996, 110-115. 
unterbreitete, wobei er von insgesamt etwa 600 zu verlegenden geisteskranken Südtirolern ausging. Für diesen Transport, der zur Brennergrenze gehen sollte, hätte er bereits entsprechende Vorbereitungen getroffen. ${ }^{30}$ Greifelt war jedoch der Ansicht, der Transport solle wenn, dann direkt in die aufnehmenden deutschen Anstalten geleitet werden. ${ }^{31}$ Bereits wenige Tage später, am 23. April 1940, erkundigte der Präfekt sich bei der ADERSt in Bozen, ob bereits Weisungen von Berlin eingegangen seien, welches Ziel der Transport haben solle. ${ }^{32}$ Dies zeigt, dass zu diesem Zeitpunkt vor allem die italienische Seite an einer raschen Abschiebung der Kranken interessiert war, auf der deutschen Seite allerdings noch organisatorische Unklarheiten bestanden, die jedoch im Mai 1940 geklärt schienen. So gab Greifelt am 17. Mai 1940 bei einer Besprechung in Innsbruck sein Plazet zum Abtransport der etwa vierzig Patienten. ${ }^{33}$ Dieses Vorgehen scheint indes nicht der ursprünglichen Weisung Himmlers entsprochen zu haben, denn bei der nachfolgenden Besprechung am 20. Mai 1940 in Bozen wurde beschlossen «der ursprünglichen Weisung des Reichskommissars zufolge die Irren auf einmal, und zwar in höchstens 2 Transporten abzunehmen». ${ }^{34}$ Dies korrespondierte auch mit dem Vorgehen im Rahmen der Umsiedlung der Baltendeutschen, denn bereits im Dezember 1939 und im Januar 1940 hatten zwei große Transporte mit psychisch kranken Baltendeutschen Lettland in Richtung des Deutschen Reiches verlassen. ${ }^{35}$

In Bozen wurde weiterhin vereinbart, alle in Frage kommenden Kranken zunächst nach Pergine zu verlegen und von dort geschlossen nach Württemberg zu überstellen. Die Abwanderung sollte mittels eines Sammelpasses erfolgen. Zudem sollte der Leiter der Heilanstalt Zwiefalten vorläufig die Vormundschaft für alle Patienten übernehmen, deren freiwillige Option für Deutschland zwar eine notwendige Voraussetzung für ihre Umsiedlung hätte sein müssen, in der Realität jedoch nur in den wenigsten Fällen vorlag. Der Bericht über den Transport bemerkt zur Geschäftsfähigkeit und damit zur Zulassung zur Option lediglich:

30 Tatsächlich hatten sich verschiedene Dienststellen bereits an die Heilanstalt Pergine gewandt, mit der Bitte eine Liste der optionsberechtigten Patienten aufzustellen und den Patienten bei der Option «behilflich» zu sein. Vgl. Arreghini 1995.

31 Vgl. Stuhlpfarrer 1985, 518.

32 Aktenvermerk Luig (ADERSt Bozen) betr. Herausnahme der Irren vom 25.4.1940, BA Berlin, R 49/2146, unpag.

33 Protokoll der Besprechung im Gasthof Greifen (Bozen) am 16.4.1940, undat., BA Berlin, R 49/2114, unpag.

34 Aktenvermerk Luig betr. Herausnahme der Irren, 20.5.1940, BA Berlin, R 49/2146, unpag.

$35 \mathrm{Vgl}$. Schreiben der NSDAP Kreisleitung Swinemünde an die Volksdeutsche Mittelstelle Posen betr. Krankentransporte ins Reich vom 23.2.1940, APP, Vomi, 123, Bl. 43. Vgl. auch Fiebrandt 2012. 
Die Kranken waren natürlich nicht dazu in der Lage, ihre Stimme selbst für Deutschland abzugeben; für einen Teil von ihnen wurde dies von den Angehörigen erledigt; bei den anderen liess [sic!] sich die deutsche Volkszugehörigkeit aus dem Geburtsort und dem Namen nachweisen. ${ }^{36}$

De jure wäre ein solches summarisches Vorgehen nicht zulässig gewesen, da die Optionsentscheidung eine individuelle und freie sein sollte. ${ }^{37}$ Hinzu kam, dass dem italienischen Recht nach die Aufnahme in einer psychiatrischen Anstalt mit dem Verlust der bürgerlichen Rechte, und somit auch des Wahlrechts verbunden war - die Patienten wären demnach pro forma von der Option ausgeschlossen gewesen. ${ }^{38}$ Das Optionsrecht übten schließlich, ohne dass dafür eine rechtsgültige Vereinbarung zwischen dem Deutschen Reich und Italien getroffen worden wäre, in einigen wenigen Fällen Verwandte, in den meisten jedoch die Verwaltung der Provinz Bozen aus. ${ }^{39}$ Da jedoch von keiner Seite Einwände gegen dieses Vorgehen vorgebracht wurden, begannen noch am 20. Mai 1940 die konkreten Transportvorbereitungen. Am 21. Mai 1940 fanden in Pergine zusammen mit dem Direktor Dr. Alberto Rezza die abschließenden Gespräche statt. ${ }^{40}$

Maßgeblichen Anteil an der Organisation und Durchführung des Transportes hatten neben dem Bozener Präfekten vor allem Dr. Walther Simek, als Beauftragter der Auslandsabteilung der Reichsärztekammer (RÄK), und die Mitarbeiter Dr. Reinhold Wetjen und Dr. Hermann Pedoth von der ADERSt in Bozen. Die RÄK war bereits bei der Umsiedlung der psychisch kranken Baltendeutschen in Erscheinung getreten, nachdem der Reichsgesundheitsführer Leonardo Conti, dem von Himmler in seiner Funktion als RKF die gesundheitliche Betreuung der volksdeutschen Umsiedler übertragen worden war, die Auslandsabteilung der RÄK mit der Durchführung der Krankentransporte betraut hatte und einen Beauftragten für die ge-

36 Bericht über den Transport der geisteskranken volksdeutschen Optanten aus der Anstalt Pergine bei Trient nach Zwiefalten (Württemberg) vom 18.6.1940, Dr. Simek, BA Berlin, R 49/2265, S. 2.

37 Vgl. Richtlinien für die Rückwanderung der Reichsdeutschen und Umsiedlung der Volksdeutschen aus dem Alto Adige vom 21.10.1939, in: Handausgabe der Umsiedlungs-Bestimmungen für die deutschen Optanten 1940, 6-12.

$38 \mathrm{Vgl}$. Hinterhuber 1995, 58f.

39 Arreghini ermittelte anhand der 124 noch in Pergine vorhandenen Optionsunterlagen, dass lediglich in 2 Fällen ein offiziell bestellter Vormund für Patienten optierte, in den anderen Fällen stellten Angehörige und vor allem die Verwaltung Bozen, die ADERSt oder Gemeinden einen Optionsantrag, der in den meisten Fällen von Angehörigen unterzeichnet wurde. Vgl. Arreghini 1995. Siehe dazu beispielsweise den Abwanderungsantrag von Maria L., der am 13.3.1940 von ihrer Mutter unterschrieben wurde, ohne dass diese als offizieller Vormund der volljährigen Tochter erscheint. Zudem findet sich in der Optionsakte von Maria L. der Aktenvermerk «Antragsteller nicht geschäftsfähig». TLA, Reichsstatthalter, DUS, Optionsakte Maria L., Kennnummer 227146.

40 Bericht über den Transport Pergine - Zwiefalten, BA Berlin, R 49/2265, S. 3-5. 
sundheitliche Betreuung der volksdeutschen Umsiedler ernannt hatte. ${ }^{41}$ Aber nicht nur institutionell sondern auch in Bezug auf die fachärztliche Betreuung des Krankentransportes werden Parallelen zwischen dem Transport der Südtiroler und der Baltendeutschen sichtbar. So griff man zum einen auf einheimische Psychiater, im Falle Pergines auf den Direktor Dr. Rezza, und zum anderen auf einen deutschen Nervenarzt, in beiden Fällen Dr. Wilhelm Schneider von den Wittenauer Heilstätten Berlin, als Transportbegleitung zurück. ${ }^{42}$ Das Begleitpersonal sollte aus Schwestern und Pflegern aus Pergine bestehen. Interessant ist hierbei, dass die Innsbrucker DUS, die quasi alle Südtiroler im Zuge der Einbürgerung durchlaufen mussten und die u.a. auch für die Unterbringung alter und kranker Südtiroler im Reichsgebiet zuständig war, nicht in die Vorbereitung und Durchführung des Transportes involviert war, sondern dieser Transport ausschließlich in den Händen der ADERSt und RÄK lag. Möglicherweise war eine Einbürgerung der Südtiroler Patienten durch die DUS zu keinem Zeitpunkt beabsichtigt.

Die Verlegung der Südtiroler Patienten erfolgte schließlich am 26. Mai 1940, nachdem erst zwei Tage zuvor die letzten Patienten aus den Anstalten Stadlhof, Gemona, Nomi und Udine, die für die Weiterverlegung vorgesehen waren, in Pergine eingetroffen waren. Der Sonderzug für die 299 Patienten aus Pergine, welcher von der italienischen Seite zur Verfügung gestellt wurde, umfasste 5 Waggons zweiter Klasse, einen Rot-Kreuz-Wagen und einen Gepäckwagen. Um 1.15 Uhr wurde mit der «Einwaggonierung» der Patienten begonnen und um «8.45 abends» traf der Zug in Zwiefaltendorf ein, wo er vom Direktor der Anstalt Zwiefalten, Dr. Alfons Stegmann ${ }^{43}$ und, wie es hieß, der «nötige[n] Anzahl von Krankenwärtern und -wärterinnen» in Empfang genommen wurde. Gegen «10.30 abends war die Auswaggonierung» beendet. ${ }^{44}$ Simek resümierte in seinem Bericht schließlich: «Nur durch die vorzügliche Zusammenarbeit zwischen den italienischen und deutschen Stellen war es möglich gewesen, den Transport so schnell und reibungslos durchzuführen.» Diese Einschätzung macht nochmals deutlich, dass die Überführung psychisch Kranker aus Südtirol im Interesse der italienischen wie auch der deutschen Seite lag und eine aus Sicht der Akteure erfolgreiche

41 Hellmut, Haubold übernahm im März 1940 die Leitung der Auslandsabteilung der RÄK, bereits seit Ende 1939 war er zudem zum Beauftragten des Reichsgesundheitsführers für die gesundheitliche Betreuung der volksdeutschen Umsiedler avanciert. Vgl. diesbezüglichen Schriftverkehr in BA Berlin, R 1501/3802.

42 Vgl. Bericht über den Transport Pergine-Zwiefalten, BA Berlin, R 49/2265 sowie Bericht über den Geisteskrankentransport Riga-Arnsdorf vom 18.12.1939, APP, Vomi, 123, Bl. 112-118. Für Informationen zu Schneider danken wir Thomas Beddies, Berlin, sehr herzlich.

43 Zu Stegmanns Rolle vergleiche auch Rexer 1996, 34.

$44 \mathrm{Vgl}$. Bericht über den Transport Pergine-Zwiefalten, BA Berlin, R 49/2265, S. $12,14$. 
und rasche Durchführung, ohne Rücksicht auf die Patienten selbst, auch hinsichtlich der Beziehungen der Bündnispartner zueinander erwartet wurde.

So reibungslos wie von Simek in seinem für die beteiligten Dienststellen angefertigten, mit Fotos illustrierten Bericht beschrieben, verlief der Transport respektive die «Auswaggonierung» allerdings nicht. Der Zwiefalter Direktor Dr. Stegmann führte in seinem wenige Tage nach dem Eintreffen der Südtiroler angefertigten Bericht vielmehr aus: «Das Ausladen und die Beförderung der Kranken bereitete erhebliche Schwierigkeiten und zwar insofern als die grössere [sic!] Anzahl derselben hinfällig ist und z.T. überaus erregt waren.» ${ }^{45}$ Dafür, dass in diesem Punkt eher der Einschätzung Stegmanns, als dem lapidaren Bericht Simeks Glauben zu schenken ist, spricht ein Bericht des Medizinalrates Dr. Alois Bischoff, der 1940 die Heilanstalt Weissenau leitete, in die ein Teil der Patienten aus Südtirol weiterverlegt wurde. Diesem Bericht nach befanden sich offenbar alle der 75 in der Weissenau aufgenommenen Patienten in einem schlechten Allgemeinzustand. ${ }^{46}$

Dies war angesichts der Strapazen der langen Fahrt, der Ankunft in einer fremden Umgebung und der schweren Erkrankung ${ }^{47}$ der meisten der Patienten keineswegs überraschend, wurde von den Verantwortlichen allerdings billigend in Kauf genommen und war Simek keine Bemerkung wert.

Die Patienten wurden schließlich auf den Abteilungen untergebracht. Für 110 männliche Kranke standen allerdings nur Matratzen im sog. Gastbau zur Verfügung. ${ }^{48}$ Die Verständigung mit den Kranken gestaltete sich aufgrund der Sprachbarrieren schwierig, da nicht alle Südtiroler Patienten der deutschen Sprache mächtig waren. Wie übereilt der Transport stattfand und wie wenig die Weiterbehandlung der Patienten vorbereitet worden war, zeigt auch die Tatsache, dass die Krankenakten bzw. Auszüge ausschließlich in italienischer Sprache abgefasst und nicht übersetzt worden waren. Das

45 Bericht Stegmanns über die Übernahme von deutschstämmigen Geisteskranken aus oberitalienischen Heilanstalten vom 29.5.1940, adressiert an das Württembergische Ministerium des Innern in Stuttgart, Archiv des Zentrums für Psychiatrie (ZfP) Südwürttemberg, Standort Münsterklinik Zwiefalten, Ordner Südtiroler.

46 Vgl. Jahresbericht der Heilanstalt Weissenau 1940 vom 2.5.1941 (Dr. Bischoff), Archiv des ZfP Südwürttemberg, Standort Ravensburg-Weissenau. Ohne dass dies zum gegenwärtigen Zeitpunkt hinreichend interpretiert werden kann, werden auch in anderen Rubriken dieses Berichts, bspw. hinsichtlich der Beschäftigung der Patienten, die Südtiroler statistisch getrennt aufgeführt oder jedenfalls erkennbar getrennt gelistet.

47 Stegmann formulierte im zeitgenössischen Duktus, dass «mindestens 80\% sämtlicher hierher verlegten Kranken [...] schizophrene Endzustände, hinfällige Paralytiker sowie Epileptiker mit starken Wesensveränderungen» seien.Vgl. Bericht Stegmanns über die Übernahme von deutschstämmigen Geisteskranken aus oberitalienischen Heilanstalten vom 29.5.1940, Archiv des ZfP Südwürttemberg, Standort Münsterklinik Zwiefalten, Ordner Südtiroler.

48 Einem Gebäudeflügel der ehemaligen Klosteranlage, in der die ehemaligen Gasträume des Abtes und im Krankenhausbetrieb von 1940 die Stationen für Männer untergebracht waren. 
scheinbar wichtigste für beide Seiten war der erfolgte Transfer, der mit dem Ausbürgerungsschein auch verwaltungstechnisch seinen Abschluss fand. ${ }^{49}$ Für das Begleitpersonal fand anschließend eine Besichtigungsfahrt nach Stuttgart und München statt, wo u.a. das Kaiser-Wilhelm-Institut besucht wurde, ${ }^{50}$ bevor es am 29. Mai 1940 nach Italien zurückkehrte.

Bereits nach wenigen Tagen waren die ersten Todesfälle unter den Südtiroler Kranken zu verzeichnen. Bis Ende Juli 1940, also innerhalb von etwa zwei Monaten, waren bereits 14 Patienten (6 Männer, 8 Frauen) verstorben. ${ }^{51}$ Am 6. Juli 1940 wurden, wie bereits erwähnt, 75 männliche Patienten in die Heilanstalt Weissenau verlegt, am 19. Oktober 1940 weitere 25 Patienten (12 Männer, 13 Frauen) in die Heilanstalt Schussenried..$^{52}$ Auch in der Weissenau war die Sterblichkeit hoch, wie dem Bericht für das Jahr 1940 zu entnehmen ist. Darin hieß es:

Die Sterblichkeit auf der Männerseite war in diesem Jahr erheblich höher als auf der Frauenseite [...], gegenüber dem vorigen Jahr, wo das Verhältnis umgekehrt war. Der Grund liegt darin, dass eine Anzahl hinfälliger Frauen 1940 starb, während die entsprechenden hinfälligen Männer 1941 nachkamen. Sehr wesentlich wird die erhöhte männliche Sterblichkeit durch die Südtiroler bedingt, 10 von 24 gestorbenen Männern sind Südtiroler. Von den 75 hierher verlegten Südtirolern waren am 31.12.41 also in eineinhalb Jahren ihres Hierseins 14 gestorben, das sind 18\%. Diese hohe Sterblichkeit hat ihren Grund zum Teil darin, dass die von Zwiefalten hierher verlegten Südtiroler die Auswahl der Hinfälligsten darstellten. ${ }^{53}$

Die hohe Sterblichkeit dürfte allerdings auch noch andere Ursachen gehabt haben. So könnte auch die kriegsbedingt immer stärkere Inanspruchnahme von Anstaltsräumen für andere Zwecke, so zum Beispiel ein Militärlazarett mit ca. 300 Betten im ehemaligen Klostergebäude, sowie Unterbringungsmöglichkeiten für Zwangsarbeiter im Neubau (der 1930er Jahre), und die

49 Vgl. Bericht Stegmanns über die Übernahme von deutschstämmigen Geisteskranken aus oberitalienischen Heilanstalten vom 29.5.1940, ZfP Südwürttemberg, Standort Münsterklinik Zwiefalten, Ordner Südtiroler.

50 Das gesamte Begleitpersonal besuchte am 27.5.1940 Stuttgart, am 28.5.1940 München. Nach einer Fahrt durch München wurde die Kriegswochenschau besucht. Anschließend fand für die Schwestern eine Führung durch die Kirchen Münchens statt, die Ärzte waren bei Dr. Harrfelt, dem Leiter der Ärztekammer Bayerns, eingeladen. Daran schloss sich ein Besuch des Kaiser-Wilhelm-Instituts, vermutlich dasjenige für Psychiatrie an. Nach einem gemeinsamen Kameradschaftsabend erfolgte am nächsten Morgen die Rückreise. Vgl. Bericht über den Transport Pergine - Zwiefalten, BA Berlin, R 49/226.

51 Vgl. Hauptbuch der Heilanstalt Zwiefalten, Männer-Abteilung, 1940-1948, sowie Hauptbuch der Frauen-Abteilung 1939-1949, Archiv des ZfP Südwürttemberg, Standort Münsterklinik Zwiefalten.

52 Vgl. Hauptbücher Zwiefalten sowie Ergänzung zum Aufnahmebuch Weissenau, Archiv ZfP Südwürttemberg, Standort Ravensburg-Weissenau, und Aufnahmebücher Männer (19181949) und Frauen (1927-1945) Schussenried, Archiv ZfP Südwürttemberg, Standort Bad Schussenried. Eine namentliche Liste der aus Pergine nach Zwiefalten und von dort weiterverlegten Patienten ist zudem zu finden im TLA, Reichsstatthalter, DUS, 57, unpag.

53 Jahresbericht der Heilanstalt Weissenau 1940 vom 5.3.1942 (Dr. Bischoff), ZfP Südwürttemberg, Standort Ravensburg-Weissenau, hier Punkt 1 «Allgemeine Krankenbewegung». 
damit verbundene Zusammendrängung der Patienten im verbliebenen Anstaltsraum eine Rolle gespielt haben. ${ }^{54}$ Auch die mangelhafte Versorgung der Patienten mit Medikamenten und Nahrungsmitteln, die, wie Faulstich für badische Anstalten gezeigt hat, ${ }^{55}$ auch hier möglicherweise nicht allein Folge eines allgemeinen Mangels der Kriegszeit gewesen ist, sondern auch sozialutilitaristischen Zielsetzungen folgte, mag zu der hohen Sterblichkeit geführt haben. ${ }^{56}$ Der im Jahresbericht von 1941 erwähnte Personalmangel, der durch die Einziehung männlicher Pfleger und Ärzte zum Kriegsdienst oder ihre Tätigkeit in Industrieheimen bedingt war, wirkte sich auf die Gesamtsituation sicher ebenfalls nicht förderlich aus. ${ }^{57}$ Dies dürfte letztlich für alle der drei südwürttembergischen Anstalten, in die Südtiroler gelangten, gelten, auch wenn sich die Sterblichkeit zwischen den Anstalten im Einzelnen durchaus unterschied. ${ }^{58}$ Dabei war es im Anschluss an den großen Transport vom Mai 1940 nach Zwiefalten in erster Linie die Heilanstalt Schussenried, in die Südtiroler in der Folgezeit verlegt wurden.

\section{Die Einweisungen Südtiroler Kranker in die Tiroler Heilanstalt Hall und ihre Verlegung nach Schussenried}

Die Verlegung von weiteren psychisch kranken und geistig behinderten Südtirolern nach Württemberg erfolgte unter anderen Vorzeichen als die der Perginer Patienten. Zum einen handelte es sich hier um bislang nicht hospitalisierte Südtiroler, die in ihren Familien lebten und die erst im Zuge der Umsiedlung und durch deren Erfassungsmechanismen psychiatrisiert wurden. ${ }^{59} \mathrm{Zum}$ anderen wurde nun den Optionsunterlagen große Beachtung geschenkt und auch die ADERSt und die DUS traten deutlicher in Erscheinung.

54 Siehe hierzu Kretschmer 1983, 337-354, hier v.a. 344-347. In der Anstalt Weissenau sollte ein mehrere Hundert Betten umfassendes Arbeiterheim eingerichtet werden. Ein Teil der Gebäude war wie oben erwähnt zur Unterbringung sog. Zwangsarbeiter vorgesehen, die zu Arbeiten in der kriegswichtigen Industrie im nahe gelegenen Friedrichshafen gezwungen waren. Zur Fremdnutzung von Anstaltsgebäuden vgl. auch Süß 2003.

55 Faulstich 1998, hier v.a. 301-305, 343-377 (v.a. S. 351-355).

56 Vgl. dazu auch Süß 2003.

57 Zur Situation der Weissenau in der Zeit zwischen 1933 und 1945 siehe auch Steinert 1985, 163-202 sowie: Kretschmer 1983, hier v.a. 344-347.

58 Vgl. u.a. May 1996 b.

59 Es handelte es sich hier überwiegend um Familienmitglieder, deren Krankheit erst im Zuge der Umsiedlung aktenkundig wurde bzw. als therapiebedürftig eingeschätzt wurde, daraufhin erfolgte die Psychiatrisierung. Einige wenige waren zuvor bereits kurzzeitig in Anstaltspflege gewesen, aber wieder nach Hause entlassen worden. Patienten, die in sog. Psychiatrischer Familienpflege versorgt wurden, werden nicht explizit erwähnt, könnten sich aber durchaus auch darunter befunden haben. 


\section{Die Erfassungsmechanismen der ADERSt in Südtirol und der DUS in Innsbruck}

Den administrativen Ausgangspunkt für die Umsiedlung stellte für jeden Südtiroler Umsiedler laut den Vereinbarungen zwischen dem Deutschen Reich und Italien vom 23. Juni 1939 die Einreichung eines Abwanderungsantrages bei der für seinen Wohnort zuständigen ADERSt-Dienststelle dar. In diesem Abwanderungsantrag erklärte der Antragsteller «sämtliche Angaben über [sich] und [seine] Familienangehörigen der Wahrheit entsprechend gemacht zu haben» insbesondere was die «arische Abstammung» anbelangte. ${ }^{60}$ Auf der Rückseite des Antrages wurden schließlich neben Personenstandsangaben auch Strafen bzw. anhängige Strafverfahren und «dauernde körperliche Leiden und ansteckende Krankheiten» erfragt. Auch wenn diese Formulierung noch relativ vage gefasst war, und erst in nachfolgenden Umsiedlungsaktionen ein differenziertes System der erbbiologischen Erfassung etabliert wurde, zeigt sich darin doch der Wille zur «totalen〉 Erfassung des einzelnen Umsiedlers, vor allem auch hinsichtlich «unerwünschten Bevölkerungszuwachses». So erscheinen unter diesem Punkt schließlich auch «Diagnosen» wie «geistesminderwertig» oder «psychisch abnormal», ${ }^{61}$ die deutlich machen, dass auch die Umsiedlung zu einem Aktionsfeld rassenhygienischer Überzeugungen wurde.

Einem Erlass des Reichsministeriums des Innern (RMdI) vom 3. August 1939 zufolge sollte bei «Berücksichtigung der Gesamtverhältnisse und des besonderen optionsähnlichen Charakters der Gesamtaktion [...] von der Beibringung besonderer ärztlicher Bescheinigungen über die gesundheitliche und erbbiologische Eignung der Antragsteller und ihrer Familienangehörigen abgesehen werden», ${ }^{62}$ indirekt wurde diese Eignung allerdings im Abwanderungsantrag durch die Kategorie «Krankheiten» geprüft. Im «Verdachtsfall» wurde durch die regional zuständige ADERSt-Stelle ein ärztliches Gutachten eingeholt und der Abtransport mittels Krankenwagen vorbereitet. ${ }^{63}$ In allen ADERSt-Zweigstellen und der Hauptstelle in Bozen

60 Abwanderungsantrag von Rosa Z. vom 11.4.1940 (ADERSt Meran), TLA, Reichsstatthalter, DUS, Optionsakte Rosa Z., Kennnummer 230 302. Die Optionsunterlagen der umgesiedelten Südtiroler befinden sich nahezu vollständig im Tiroler Landesarchiv in Innsbruck.

61 Vgl.Abwanderungsantrag Anna T.vom 20.2.1940 (ADERSt Meran), TLA, Reichsstatthalter, DUS, Optionsakte Anna T., Kennnr. 223 624, Abwanderungsantrag Barbara L. vom 16.3.1940 (ADERSt Bruneck), ebd., Optionsakte Barbara L., Kennnr. 412475.

62 Schnellbrief des RMdI an den Landeshauptmann in Tirol betr. Einbürgerung von Volksdeutschen aus Italien, 3.8.1939, S. 4, BA Berlin, R 49/1173, unpag.

63 Vgl.z.B. Optionsakte Rosa Z., Kennnr. 230 302, TLA, Reichsstatthalter, DUS oder Optionsakte Maria P., Kennnr. 304 826, ebd. 
war dafür die Abteilung IV «Soziales» zuständig. ${ }^{64}$ In Vorbereitung des Abtransportes setzte sich diese mit der DUS, Abteilung IX «Betreuung», ${ }^{65}$ in Innsbruck in Verbindung und teilte beispielsweise mit, dass «der Geisteskranke am Montag den 3. März 1941 mittels Auto nach Innsbruck gebracht wird», verbunden mit der Bitte «um Kenntnisnahme und weitere Veranlassung». ${ }^{66}$ In dem zitierten Fall von Josef W. aus Platt bedeutete dies die Einweisung in die Heil- und Pflegeanstalt Hall in Tirol. Er war jedoch kein Einzelfall. In vielen weiteren Fällen wurde die Einweisung in eine Heilanstalt, insbesondere in die Heilanstalt Hall, von der ADERSt empfohlen, und von der Abteilung IX der DUS in Innsbruck veranlasst. Anhand des Aufnahmebuches lassen sich für die Heilanstalt Hall für die Jahre 1940 bis 1943 über 250 Einweisungen von Südtirolern durch die DUS nachweisen. ${ }^{67}$

Gleichzeitig begann die ADERSt gezielt bestimmte Bevölkerungsgruppen wie Taubstumme und «Kretine», die sich im Abwanderungsgebiet befanden, in separaten Aufstellungen zu erfassen. Im Falle der «Kretine» konnte auf ein bereits etabliertes Netz von Beratungsstellen für «kropfbehaftete und kretinöse» Kinder zurückgegriffen werden, welches von Dr. Rudolf Jungwirth, einem Meraner Kinderarzt, bereits seit den 1930er Jahren aufgebaut worden war. Die von Jungwirth der ADERSt gemeldeten «kretinösen» Kinder und Erwachsenen wurden während der Umsiedlung gesondert erfasst und zum Teil zur weiteren Beobachtung in die Psychiatrisch-Neurologische Klinik in Innsbruck eingewiesen. Zumeist erfolgte anschließend die Verlegung nach Hall oder in das St. Josefs-Institut in Mils. ${ }^{68}$

Die Erfassung der Kranken stützte sich demnach wesentlich auf regionale Vorarbeiten und die Ermittlungen der ADERSt. Es gab aber auch Fälle, in

64 Vgl. Organisationsplan der ADERSt, BA Berlin, R 49/2230.

65 Die Abteilung IX der DUS war u.a. befasst mit der Betreuung von Kindern und Jugendlichen durch die NSV, die Unterbringung dieser in «Jugendheimstätten», Adoptionsfragen, Unterbringung Alter und Kranker in Altersheimen und der ärztlichen Betreuung der Südtiroler in Innsbruck. Die Unterabteilung «ärztliche Betreuung» unter Leitung von Dr. [Vorname unbekannt] Helm und Dr. [Vorname unbekannt] Springer veranlasste die Einweisungen in Heilanstalten. Vgl. Organisationsplan der DUS, BA Berlin, R 49/2109 sowie in zusammengefasster Form: Alexander/Lechner/Leidlmair 1993, 307-310.

66 ADERSt Zweigstelle Meran, Abt. IV an DUS Innsbruck betr. W. Josef, 19.2.1941, TLA, Reichsstatthalter, DUS, Optionsakte Josef W., Kennnr. 232937.

67 Vgl. Aufnahmebuch der Heilanstalt Hall 1938-45, Historisches Archiv Psychiatrisches Krankenhaus Hall i. Tirol. An dieser Stelle sei dem Psychiatrischen Krankenhaus in Hall, vor allem Oliver Seifert, gedankt, der unkompliziert eine Einsicht in die betreffenden Bestände ermöglichte.

68 Zur Tätigkeit Jungwirths vgl. Sammlung Scharfetter, Universitätsarchiv Innsbruck. Wir danken Dr. Peter Goller vom Universitätsarchiv Innsbruck, der den Zugang zu diesem Bestand möglich machte. Zur Einbeziehung des St. Josef-Instituts in Mils und der dort untergebrachten Südtiroler Kinder in die sog. Kinder- und Jugendlichen-Euthanasie vgl. Karlegger 2006. 
denen zunächst eine reguläre Umsiedlung in die Wege geleitet wurde, und erst im Zuge des Einbürgerungsprocederes ein fachärztliches Gutachten eingeholt wurde und eine Einweisung nach Hall erfolgte. Im Rahmen der Einbürgerung, welche durch die DUS in Innsbruck auf Grundlage der Unterlagen der ADERSt vollzogen wurde, war es nämlich für jeden Südtiroler obligatorisch, verschiedene Dienststellen der DUS, unter ihnen auch die ärztliche Betreuungsstelle, zu durchlaufen. ${ }^{69}$ Erbrachte die Untersuchung einen psychiatrisch auffälligen Befund, wandte sich die ärztliche Betreuungsstelle an die Psychiatrisch-Neurologische Klinik Innsbruck, die wiederum den Optanten zwecks einer fachärztlichen Untersuchung aufnahm. So wurde beispielsweise Filomena C., in deren Abwanderungsantrag keinerlei Krankheiten angegeben waren, durch die ärztliche Betreuungsstelle in Innsbruck der Klinik mit der Bitte um Untersuchung und Übersendung eines Befunds überwiesen. Nach etwa drei Wochen wurde sie von dort in die Heil- und Pflegeanstalt Hall verlegt. ${ }^{70}$ Über 100 Südtiroler Patienten wurden allein in den Jahren 1940 bis 1942 wie Filomena C. aus der PsychiatrischNeurologischen Klinik in die Heilanstalt Hall überwiesen. ${ }^{71}$

Innerhalb der Umsiedlung kam der DUS somit eine Art Schleusenfunktion zu, denn alle Umsiedler, die für Deutschland optiert hatten, mussten das Einbürgerungsprocedere der DUS absolvieren. ${ }^{72}$ Dabei kam vor allem der ärztlichen Betreuungsstelle die entscheidende Aufgabe der Selektion zu, denn ihr Urteil entschied nicht selten darüber, wer tatsächlich eingebürgert werden sollte, und somit Teil der imaginären Volksgemeinschaft wurde, oder wer lediglich von Italien übernommen werden sollte, ohne jedoch die deutsche Staatsbürgerschaft zu erhalten. Galt anfangs der 1939 vereinbarte Grundsatz «Wer optionsberechtigt ist, muß eingebürgert werden, wer nicht optionsberechtigt ist, kann eingebürgert werden», ${ }^{73}$ so ordnete die Dienststelle des RKF im Juni 1940 an, dass «Volksdeutsche[,] die wegen ihres geis-

69 Jeder Umsiedler erhielt bei seinem Eintreffen in Innsbruck eine «Kontrollkarte» auf der die zu besuchenden Dienststellen vermerkt waren und nach Besuch derselben ein Stempel den Besuch quittierte. Vgl. z.B. Kontrollkarte Filomena C., TLA, Reichsstatthalter, DUS, Optionsakte Filomena C., Kennnr. 210644.

70 TLA, Reichsstatthalter, DUS, Optionsakte Filomena C., Kennnr. 210 644. Vgl. auch Krankenakte Filomena C. der Heilanstalt Hall, Historisches Archiv Psychiatrisches Krankenhaus Hall i. Tirol.

71 Vgl. Aufnahmebuch Hall 1938-45, Historisches Archiv Psychiatrisches Krankenhaus Hall i. Tirol. Die Zahl bezieht sich auf die Patienten, deren letzter Wohnort vor der Einweisung in die Klinik in Südtirol lag, die also in unmittelbarem Zusammenhang mit der Umsiedlung eingewiesen wurden.

72 Vgl. Alexander 1993, 49-54.

73 Abschrift eines Schreibens der DUS an RKF betr. Optionsberechtigung und Einbürgerungsanspruch, 26.7.1940, BA Berlin, R 49/1173, unpag. 
tigen oder körperlichen Gesundheitszustandes keinen erwünschten Bevölkerungszuwachs darstellen, [...] zwar ebenso wie die Vorbestraften oder die in Haft befindlichen Verbrecher [....] deutscherseits übernommen werden» müssten, es jedoch «keine Veranlassung [gäbe] diese durch Einbürgerung auch förmlich in den Verband des Reiches aufzunehmen». ${ }^{74}$

Bei der Entscheidung, wer als «unerwünschter Bevölkerungszuwachs» zu betrachten, und damit nicht einzubürgern sei, griff die ADERSt, wie auch die DUS auf regionale Initiativen und Vorarbeiten, wie z.B. die Jungwirths zurück. Die Konsequenzen für die Betroffenen waren dabei nicht nur in Bezug auf ihre Staatsbürgerschaft gravierend, sondern auch im Hinblick auf eine mögliche Einbeziehung in die NS-«Euthanasie», in deren Aktionsradius sie durch die Einweisung in eine Heilanstalt gelangten. Dieses Risiko bestand für die Südtiroler sowohl in der Heilanstalt Hall als auch in der Heilanstalt Schussenried, wohin die meisten Südtiroler bis 1942 verlegt wurden.

\section{Die Verlegungen aus Hall und Innsbruck nach Schussenried}

Im Zuge der Umsiedlung gelangten über 700 Südtiroler Kranke ${ }^{75}$ nach Hall, die wie bereits beschrieben über die DUS oder die Psychiatrisch-Neurologische Klinik eingewiesen worden waren. Die ersten Einweisungen von Südtirolern in Hall fielen dabei mit der Vorbereitung und dem Beginn der systematischen Ermordung psychisch kranker und geistig behinderter Menschen zusammen. Nachdem im Januar 1940 die «Aktion T4» in Brandenburg, später Grafeneck, aufgenommen worden war, setzte sie mit der «Inbetriebnahme» der Tötungsanstalt Hartheim im Mai 1940 auch in der sog. Ostmark ein. ${ }^{76}$ Im Juni 1940 begann eine Gutachterkommission der «T4» verschiedene Anstalten zu besuchen, Ende August/Anfang September traf eine solche auch in der Heilanstalt Hall ein. Anhand der Krankenakten füllten die Kommissionsmitglieder die Meldebögen aus, ohne die Patienten untersucht zu haben. ${ }^{77}$ Im Dezember 1940 trafen schließlich die Transportlisten in Hall ein, die die Verlegung von 290 Patienten, was in etwa der Hälfte aller in Hall untergebrachten Patienten entsprach, vorsahen. Darunter be-

74 RKF an DUS, 10.6.1940, ebd., unpag.

75 Hinterhuber $1995,81$.

76 Vgl. Kepplinger 2008.

77 Vgl. Aussage Ernst v. Klebelsberg vor dem Landesgericht Innsbruck am 5.8.1948, TLA, Landesgericht Innsbruck (LG Ibk.), 10 Vr 4740/47, Bd. 1, unpag. sowie allgemein zu Hall Seifert 2008. 
fanden sich auch einige Südtiroler Patienten. ${ }^{78}$ Nach Intervention des Leiters der Heilanstalt Dr. Ernst Klebelsberg wurden schließlich 106 Patienten von der Verlegung ausgenommen. Zurückgestellt wurden vor allem arbeitsfähige Patienten, Patienten mit italienischer Staatsangehörigkeit und Patienten, die sich nicht mehr in Hall befanden und hinter deren Namen auf der Rückstellungsliste «überstellt and. Anst.» vermerkt war. ${ }^{79}$ Wie sich anhand der Namen überprüfen ließ, verbarg sich dahinter u.a. die Verlegung der Südtiroler nach Schussenried am 1. November 1940. Nachweislich fünf Südtiroler Patientinnen, die überwiegend erst im Sommer 1940 in Hall eingewiesen worden waren und sich zuvor nicht in Anstaltsbehandlung befunden hatten, waren von den «T4»-Gutachtern zur Tötung vorgesehen worden und sind möglicherweise nur durch ihre vorherige Verlegung nach Schussenried nicht in Hartheim Opfer der «Euthanasie» geworden. ${ }^{80}$ Sie waren also keineswegs von vornherein aufgrund ihres Optantenstatus von der «Aktion T4» ausgenommen. Allerdings scheint im Dezember 1940 in Anwesenheit des Leiters der Heilanstalt Hall vereinbart worden zu sein, dass «die Südtiroler Rückwanderer für eine Ueberstellung nicht in Betracht» kämen. ${ }^{81}$ Die Südtiroler Patienten, die sich in Hall befanden, sollten demnach zukünftig von einer Verlegung im Rahmen der «Aktion T4» und damit der Ermordung in einer «T4»-Tötungsanstalt generell ausgenommen sein. Bei nachfolgenden Meldungen neu aufgenommener Patienten an die «T4» sollten die Südtiroler zwar auch gemeldet werden, allerdings sei «ihre Eigenschaft als Rückwanderer besonders deutlich anzugeben».82 Damit kam ihnen - ab diesem Zeitpunkt - im Vergleich zu den anderen Patienten, ebenso wie zu den ost-

78 Die Liste ist im TLA überliefert, ebenso wie die Aufstellungen über zurückgestellte Patienten, TLA, Reichsstatthalter, Abt. IIIa1, M-XI, 1 (1941). Zur Belegung vgl. Aussage Klebelsberg vom 5.8.1948, ebd., LG Ibk., 10 Vr 4740/47, Bd. 1, unpag.

79 Vgl. hierzu die Listen der zurückgestellten Patienten, TLA, Reichsstatthalter, Abt. IIIa1, M-XI, 1 (1941). Bei den Patienten mit italienischer Staatsbürgerschaft handelte es sich nicht um Optanten, sondern um Patienten, die bereits vor 1939 in Hall aufgenommen worden waren. Vgl. Aufnahmebuch Hall 1938-45, Historisches Archiv Psychiatrisches Krankenhaus Hall i. Tirol.

80 Vgl. mit der Liste der zurückgestellten Patientinnen, TLA, Reichsstatthalter, Abt. IIIa1, M-XI, 1 (1941) sowie Aufnahmebuch Hall 1938-45 und Optionsakten Rosa Z., Anna T., Rosa R., Barbara L., TLA, Reichsstatthalter, DUS.

81 Wer bei dieser Besprechung außer Klebelsberg noch anwesend war, ließ sich nicht mehr rekonstruieren. Vgl. Schreiben Klebelsberg an Czermak vom 7.1.1941, ebd, Abt. IIIa1, M-XI, 1 (1941), unpag. Möglicherweise handelte es hierbei um eine direkte Reaktion auf den in der Bozener deutschsprachigen Zeitung «Volksbote» im Dezember 1940 geäußerten «furchtbaren Verdacht», dass geistig und körperlich Kranke getötet würden. Vgl. «Der Volksbote» vom 19.12.1940, BA Berlin, R 49/2258, unpag.

82 Schreiben Czermak an Klebelsberg vom 9.1.1941, TLA, RStH, Abt. IIIa1, M-XI, 1 (1941), unpag. 
und südosteuropäischen Volksdeutschen, eine Sonderrolle zu, die sich möglicherweise durch außenpolitische Erwägungen erklären lässt. ${ }^{83}$

Parallel zum ersten «T4»-Transport aus Hall wurde demnach die Verlegung der Südtiroler nach Schussenried vorbereitet. Die Notwendigkeit dazu ergab sich aus den bereits skizzierten Abwanderungsplänen und der $\mathrm{zu}$ erfüllenden Absiedlungsquote, die vor allem zur Aussiedlung «nichtbodengebundener» Bevölkerungsteile führte, die zunächst in und um Innsbruck Aufnahme finden mussten. ${ }^{84}$ Da sich unter diesen vermehrt Altersheimbewohner, Insassen von Verpflegungshäusern, aber auch psychisch kranke Südtiroler befanden, schlug sich diese Abwanderungstaktik in einer zunehmenden Belegung der Heilanstalt Hall und örtlicher Altersheime nieder. Zur vorübergehenden Unterbringung der altersschwachen Optanten hatte die DUS in Innsbruck eigens drei Hotels requiriert und sie zu Altersheimen umfunktioniert. In der Heilanstalt Hall, die ausnahmslos alle «Geisteskranken, die einer Heilanstaltspflege bedürfen», ${ }^{85}$ aufzunehmen hatte, waren bis zum August 1940 bereits 96 Südtiroler untergebracht worden. ${ }^{86}$ Wie die DUS konstatierte, sei es aber «vollkommen undenkbar, dass die Anstalten des Reichsgaues Tirol-Vorarlberg hinsichtlich ihrer Kapacität [sic!] durch die Umsiedlung Südtirol derart in Anspruch genommen werden, dass die eigenen Aufgaben des Gaufürsorgeverbandes Gefahr laufen».87 Es sei schließlich vereinbart worden, dass die «Anstalt in Hall ausdrücklich

83 Die Südtiroler wurden nach bisherigem Forschungsstand in Zwiefalten, Schussenried und Weissenau zunächst nicht in die «Aktion T4» einbezogen. May vermutete, dass die Verantwortlichen mit einem Rückgang der Abwanderungswilligkeit rechneten, der zu einem Stocken der Umsiedlung führen würde, falls man von der Ermordung der Südtiroler Patienten bzw. allein diesem Verdacht erfahren würde. Tatsächlich wurden derartige Gerüchte, die besonders deutlich im «Volksboten» artikuliert wurden, von den Optionsgegnern in Südtirol benutzt, um die Abwanderung ins Stocken zu bringen. Die Angehörigen der nach Württemberg verlegten Kranken traten zudem gehäuft an die DUS mit der Bitte heran, Auskunft über das Befinden des Kranken zu erhalten. Die Bestätigung des Tötungsverdachts hätte die Umsiedlung und damit auch die Beziehungen zum Bündnispartner Italien sicher nachhaltig gefährdet. Vgl.u.a. May 1996b, 72 sowie Karlegger 2006,46-50. Siehe auch unten.

84 Die DUS monierte beispielsweise im Dezember 1940 «[...] Leider musste ich aber in der letzten Zeit feststellen, dass es sich bei diesen Umsiedlern teilweise um nichteinsatzfähige Personen handelt und es hat den Anschein, dass diese Umsiedler ohne besonderen Zwang nur deshalb herausgenommen werden, um die tägliche Abwanderungsziffer nicht noch weiter sinken zu lassen. [...]», DUS an ADERSt, 12.12.1940, BA Berlin R 49/2109, unpag.

85 Aktenvermerk Degischer (DUS) für Dr. Springer (ärztliche Betreuung) betr. Gau-Heil- und Pflegeanstalt Hall, 13.8.1940, ebd., unpag.

86 Vgl. Schreiben der Medizinalabteilung des Reichsstatthalters an die DUS betr. Gau-Heilanstalten, 22.8.1940, ebd., unpag.

87 Aktenvermerk Degischer (DUS) für Gaufürsorgeverband betr. Gau-Heil- und Pflegeanstalt Hall, 13.8.1940, TLA, Reichsstatthalter, DUS, 56, unpag. 
eine Durchgangsstation für diese Kranke[n]», die «Zug um Zug in den Gau Württemberg, Heilanstalt Zwiefalten eingewiesen werden» sollten, sei und etwa 20 bis 25 Plätze bereitzustellen hätte. ${ }^{88}$ Der Abtransport der in Hall untergebrachten Südtiroler sollte nach Absprache mit Dr. Klebelsberg aus Hall umgehend in die Wege geleitet werden, zumal die DUS weitere 250 pflegebedürftige Personen erwartete ${ }^{89}$ Die DUS trat deshalb an die Auslandsabteilung der Reichsärztekammer, Abteilung Heime und Heilstätten, heran, ${ }^{90}$ mit der Forderung Unterbringungsmöglichkeiten zu beschaffen, und monierte, dass «das einzige auf diesem Gebiet [von Simek/RÄK] geleistete [...] die Unterbringung von anstaltsbedürftigen Geistesschwachen in Zwiefalten, Gau Württemberg» gewesen sei. ${ }^{91}$ Diese beschränkte sich auf die Unterbringung der Patienten aus Pergine, weitere Transporte nach Zwiefalten hatten bis zum August 1940 nicht stattgefunden. Im September 1940 sicherte Simek zu, einen Transport aus Hall nach Zwiefalten in die Wege zu leiten..$^{92}$ Er wandte sich daraufhin an den Leiter der Heilanstalt Zwiefalten, Dr. Stegmann, mit der Frage «ob ein derartiger Transport in nächster Zeit durchführbar ist und wohin die Kranken gebracht werden» könnten. ${ }^{93}$ Die Anfrage wurde von Zwiefalten an das Württembergische Ministerium des Innern, Ministerialrat Dr. Eugen Stähle, weitergeleitet, welcher sich offensichtlich gegen eine Unterbringung weiterer Südtiroler in Zwiefalten aussprach und stattdessen die Heilanstalt Schussenried vorschlug. Dr. Helm von der ärztlichen Betreuungsstelle der DUS setzte sich daraufhin mit Dr. Klebelsberg, Hall, in Verbindung, der von etwa 60 bis 70 zu verlegenden Südtirolern ausging. ${ }^{94}$ Letztendlich wurden am 1 . November 1940 jedoch weitaus mehr, näm-

88 Aktenvermerk Degischer (DUS) für Gaufürsorgeverband betr. Gau-Heil- und Pflegeanstalt Hall, 13.8.1940, TLA, Reichsstatthalter, DUS, 56, unpag.

89 Degischer (DUS) an RÄK, 26.9.1940, TLA, Reichsstatthalter, DUS, 56, unpag.

90 Diese mit der Unterbringung alter, pflegebedürftiger und kranker Umsiedler befasste Dienststelle koordinierte die Verteilung der kranken Umsiedler auf die einzelnen Heime und Heilanstalten und nahm eine vermittelnde Position zwischen aufnehmenden Einrichtungen des «Altreichs» und den Umsiedlungsdienststellen ein.

91 Degischer (DUS) an RÄK, 26.9.1940, TLA, Reichsstatthalter, DUS, 56, unpag.

$92 \mathrm{Vgl}$. handschriftlicher Vermerk über eine diesbezügliche Vereinbarung zwischen Dr. Simek und Dr. Helm (ärztliche Betreuung) vom 13.9.1940 auf Aktenvermerk Degischer für Dr. Helm betr. Gau-Heil- und Pflegeanstalt Hall, 13.8.1940, Aktenvermerk Degischer (DUS) für Gaufürsorgeverband betr. Gau-Heil- und Pflegeanstalt Hall, 13.8.1940, TLA, Reichsstatthalter, DUS, 56, unpag.

$93 \mathrm{Vgl}$. Simek/RÄK an Stegmann/Zwiefalten betr. Südtiroler Geisteskranke, 12.9.1940, Hauptstaatsarchiv (HStA) Stuttgart, E 151/53, Bü 423, Bl. 14. Anhand der verfügbaren Akten der DUS und des Württembergischen Innenministeriums lässt sich nicht klären, warum die Südtiroler nicht in Zwiefalten, sondern in Schussenried untergebracht wurden. Die Unterlagen der RÄK gelten als vernichtet.

94 Vgl. Dr. Helm an Simek/RÄK betr. Überstellung von Geisteskranken von Hall nach Zwiefalten, 17.9.1940, TLA Reichsstatthalter, DUS, 56, unpag. 
lich insgesamt 195 Südtiroler nach Schussenried gebracht. ${ }^{95}$ Sie stammten nicht ausschließlich aus Hall, sondern unter ihnen befanden sich auch zehn Patienten der Psychiatrisch-Neurologischen Klinik Innsbruck und 73 pflegebedürftige Südtiroler aus den von der DUS in Pflegeheime umfunktionierten Hotels «Union», «Viktoria» und «Sonnenburgerhof». 112 Patienten stammten aus Hall. ${ }^{96}$ Es handelte sich demzufolge nicht ausschließlich um psychisch kranke Südtiroler, die einer psychiatrischen Behandlung bedurften, sondern auch um altersschwache, die lediglich pflegebedürftig waren. Hier wird also an einem weiteren regionalhistorischen Beispiel deutlich, dass im Zweifelsfall eine präzise Diagnosestellung der verantwortlichen Akteure in der NS-Psychiatrie nicht von Bedeutung war: weder wurde - selbst nicht nach zeitgenössischem Kenntnisstand - genau nach neurologischer oder psychiatrischer Erkrankung unterschieden, noch stellte die Tatsache, <allein> körperbehindert zu sein, eine Sicherheit gegen die Überstellung nach Württemberg dar. Wie hier zu sehen ist, war auch eine altersbedingte und altersadäquate Pflegebedürftigkeit für die Verantwortlichen ein Kriterium für die Überstellung der betreffenden Patientinnen und Patienten in eine psychiatrische Einrichtung.

Glaubt man den Berichten über den Krankentransport, so ergaben sich außer einer etwa dreistündigen Verspätung des Zuges, welche dazu führte, dass die Ankunft in Schussenried sich verzögerte, während des Transportes keine größeren Probleme. ${ }^{97}$ Als der Zug jedoch in Schussenried eingetroffen war, mussten die Patienten in einen Sonderzug «umgeladen» werden um zur Heilanstalt gebracht zu werden. Nach Angaben des DRK-Personals wurden «die Lahmen [...] aus dem Waggon gezerrt, stiessen bald da, bald am Trittbrett an und landeten endlich nach einem schauerlichen Tragen durch den

95 Vgl. Aufnahmebücher Männer (1918-49) und Frauen (1927-45) Schussenried, ZfP Südwürttemberg, Standort Bad Schussenried. Die Transportliste der DUS umfasste allerdings 199 Namen, 3 Patienten fehlten, 1 Patientin erschien auf der Liste doppelt. Vgl. Schussenried an Württembergisches Ministerium des Innern betr. Aufnahme der Südtiroler Siechen und Irren, 5.11.1940, HStA Stuttgart, E 151/53, Bü 423, Bl. 28.

96 Vgl. Aufnahmebücher Männer (1918-49) und Frauen (1927-45) Schussenried, ZfP Südwürttemberg, Standort Bad Schussenried. Allerdings werden hier 113 Patienten aus Hall und 72 aus Innsbruck vermerkt, aus Hall sind nachweislich jedoch nur 112 Patienten verlegt worden, vermutlich ist im Aufnahmebuch Schussenried eine falsche Zuordnung erfolgt. Vgl. Aufnahmebuch Hall 1938-45, Historisches Archiv Psychiatrisches Krankenhaus Hall i.T. Vgl. weiter Namenslisten des Transports von nicht mehr einsatzfähigen Südtiroler Umsiedlern von Innsbruck nach Schussenried und Verzeichnis der in die württ. Heil- und Pflegeanstalt in Schussenried eingewiesenen Südtiroler Umsiedler vom 12.4.1941, TLA, Reichsstatthalter, DUS, 57, unpag.

97 Vgl. Tatsachenbericht zum Transport von 190 geisteskranken und siechen Südtiroler Umsiedlern nach Schussenried am 1. November 1940 (DRK-Personal), sowie Reisebericht über den Krankentransport von Innsbruck nach Schussenried am 1. November von Dr. Helm, undatiert, TLA, Reichsstatthalter, DUS, 58, unpag. 
strömenden Regen in einem eiskalten Viehwagon der Kleinbahn, wo sie auf das Stroh [...] gelegt wurden». ${ }^{98}$ Während dieses Zugwechsels, der unter unmenschlichen Bedingungen vor sich ging, kam es dazu, dass die Patienten, die zunächst nach altersschwachen und geisteskranken getrennt transportiert worden waren, «in dem Sonderzug der Lokalbahn durch das fortwährende aufgeregte Antreiben des Med. Rates [Dr. Hugo Götz] [...] schon zum Teile durcheinander [kamen]. Nach der Ankunft in der Heilanstalt es war mittlerweile finster geworden und regnete mit Strömen - vervollständigte sich das Durcheinander». ${ }^{99}$ Die Situation nach Ankunft in der Heilanstalt beschrieb Dr. Helm von der ärztlichen Betreuungsstelle der DUS folgendermaßen:

«Ich stellte fest, daß die Vorbereitungen für die Aufnahme der Kranken wohl in der Bereitstellung von leeren Stationen, nicht aber in irgendeinem organisatorischen Plan bestanden hatten. Vor allem schien es dem Personal der Anstalt einschließlich Ärzten gar nicht irgendwie klar zu sein, daß sie nicht nur 〈ruhige〉 und 〈unruhige〉 Kranke zu übernehmen hätten, sondern daß ein guter Teil der Patienten normal und von den Irren zu trennen war. Man fragte mich immer wieder, ob diese Kranken ruhig seien und hörte gar nicht darauf, daß unter den ruhigen Narren sich soundsoviele normale Sieche - sehr zu deren Entsetzen - befanden [...].

Am nächsten Tag übergab ich dem Stellvertreter des Dr. Götz, Med. Rat Dr. Morenbach, die Transportliste und die Dokumente der Kranken. Wir gingen dann durch die Stationen. Diese sehen ordentlich aus, das Pflegepersonal macht einen freundlichen Eindruck. Ich zeigte bei jeder Gelegenheit dem Arzt und den Pflegern die Siechen, die von den Irren zu trennen seien und hoffe inständig, daß diese Trennung ehestens durchgeführt wird $[\ldots] .{ }^{100}$

Dr. Götz vermerkte in seinem Bericht an das Württembergische Innenministerium lediglich, dass «die nicht gehfähigen Kranken [...] zu den Abteilungen über eine erhebliche Strecke einzeln getragen werden [mussten], wobei die völlige Dunkelheit und anhaltender Regen die Arbeit sehr stark erschwerten, zumal die Anstalt über keine ausgiebigen Vorrichtungen für solche Zwecke verfügt». ${ }^{101}$ Die Umstände des Transportes und die Tatsache,

98 Tatsachenbericht DRK TLA, Reichsstatthalter, DUS, 58, unpag. Der Bahnhof Schussenried der sog. Südbahn von Ulm über Biberach nach Friedrichshafen usw. liegt abseits der Stadt Schussenried, das Anstaltsgelände wiederum zentral im Stadtzentrum, und war seinerzeit mit einer Schmalspurbahn («Lokalbahn») versorgt.

99 Reisebericht Dr. Helm, TLA, Reichsstatthalter, DUS, 58, unpag.

100 Reisebericht Dr. Helm, TLA, Reichsstatthalter, DUS, 58, unpag.

101 Schussenried an Württembergisches Ministerium des Innern betr. Aufnahme der Südtiroler Siechen und Irren, 5.11.1940, HStA Stuttgart, E 151/53, Bü 423, Bl. $28 f$. 
dass auch nach mehreren Tagen eine «endgültige Verteilung der Ankömmlinge» noch nicht erfolgt war, ${ }^{102}$ das heißt altersschwache und psychisch kranke Patienten gemeinsam auf den Stationen untergebracht blieben, führten in der Folgezeit zu zahlreichen Protesten seitens der Angehörigen der altersschwachen Südtiroler, der DUS und der Untergebrachten selbst, und veranlassten darüber hinaus die DUS, in Schussenried vorstellig zu werden. ${ }^{103}$ Das Württembergische Innenministerium verwahrte sich gegen die Kritik mit dem Hinweis «es dürfte allgemein bekannt sein, daß Beschwerden von Geisteskranken nicht ohne weiteres ernst zu nehmen sind und auch alte sieche Personen allzu leicht zum Querulieren neigen». ${ }^{104}$ Es verbat sich jegliche Einmischung in die inneren Angelegenheiten der Anstalt Schussenried.

Im Juli 1941 teilte schließlich die RÄK mit, dass die altersschwachen Südtiroler in die Neuendettelsauer Anstalten verlegt werden sollten, nachdem diese Anstalten geräumt, die Patienten verlegt und die Anstalt als Altersheim für Südtiroler umfunktioniert worden wäre. ${ }^{105}$

Bis 1942 wurden keine weiteren Südtiroler nach Schussenried verlegt. Allerdings trafen auch weiterhin Krankentransporte in Innsbruck ein und auch die Heilanstalt Hall nahm nach wie vor Südtiroler auf. Bereits im Januar 1941 machte Dr. Helm von der ärztlichen Betreuungsstelle deshalb darauf aufmerksam, dass ein weiterer Transport aus Hall erforderlich sei. ${ }^{106}$ Statt des geforderten Weitertransportes verzeichnete Hall allerdings weitere Aufnahmen Südtiroler Patienten, und dies nicht zuletzt deshalb, weil die italienischen Heilanstalten deren Aufnahme nun geradeweg verweigerten. ${ }^{107}$

Im November 1941 wurde seitens des Württembergischen Innenministeriums und der Heilanstalt Schussenried schließlich die Übernahme von

102 Schussenried an Württembergisches Ministerium des Innern betr. Aufnahme der Südtiroler Siechen und Irren, 5.11.1940, HStA Stuttgart, E 151/53, Bü 423, Bl. 28f.

103 Am 14.11.1940 suchten Dr. Helm und Degischer von der DUS die Heilanstalt Schussenried auf, um eine räumlich getrennte Unterbringung der altersschwachen Südtiroler und eine bessere Verpflegung dieser zu erwirken, vgl. diesbezügliche Vereinbarungen im Schreiben Schussenried an DUS vom 14.11.1940, TLA, Reichsstatthalter, DUS, 58, unpag.

104 Württembergisches Ministerium des Innern an DUS betr. Heilanstalt Schussenried, 11.12.1940, TLA, Reichsstatthalter, DUS, 58, unpag.

105 Simek/RÄK an Dr. Stähle, 25.7.1941, HStA Stuttgart, E 151/53, Bü 423, Bl. 92, siehe weiter Bl. 100-103. Vgl. zur Räumung Neuendettelsaus und der damit in direkter Verbindung stehenden Unterbringung der Südtiroler: Bayerisches Hauptstaatsarchiv München (BayHStA), MInn, 79997 und 79998. Vgl. auch Müller/Siemen 1991.

106 Dr. Helm an Degischer/DUS betr. Unterbringung von Siechen, Geisteskranken und körperbehinderten Umsiedlern, 9.1.1941, TLA, Reichsstatthalter, DUS, 56, unpag.

107 Vgl. ADERSt an RÄK, Referat Heime und Heilstätten betr. Freiplätze für Geisteskranke in der Heilanstalt Schussenried, 15.9.1941 sowie ADERSt an Gauleiter Tirol und Voralberg betr. Irrentransport am 20.12.1941, 12.12.1941, BA Berlin, R 49/922, unpag. Sowie Aufnahmebuch Hall 1938-45, Historisches Archiv Psychiatrisches Krankenhaus Hall im Tirol. 
weiteren 130 psychisch Kranken zugesagt. Im Januar 1942 wurden die ersten konkreten Vorbereitungen getroffen. ${ }^{108}$

Dabei sollten zunächst die Kranken aus Hall verlegt werden, die keinen Besuch empfangen würden bzw. deren «Verbleib in der Anstalt Hall aus sonstigen Gründen nicht tragbar» sei. ${ }^{109}$ Am 10. März 1942 wurden die ersten 17 Südtiroler aus Hall nach Schussenried verlegt, nur einen Tag später folgten ihnen 51 weitere. ${ }^{110}$

Weitere Südtiroler sollten nicht nach Württemberg gelangen, da wenige Monate später, im August 1942, das Württembergische Innenministerium der RÄK mitteilte, es könnten aufgrund der Überfüllung der Anstalten und der Nutzung dieser als Hilfslazarette «Südtiroler Kranke unmöglich mehr in württembergischen Anstalten aufgenommen werden, zumal württembergische Gebiete luftgefährdet [wären], während dies in Tirol sicherlich nicht der Fall» sei. ${ }^{111}$

Die nach Schussenried verlegten psychisch kranken Südtiroler teilten dort das Schicksal ihrer Mitpatienten, welches vor allem geprägt war von Mangelernährung, schlechter medizinischer Versorgung, Überbelegung und Krankheiten. ${ }^{112}$ Opfer der «Aktion T4» wurden die nahezu 500 nach Schussenried und Zwiefalten verlegten Südtiroler nach aktuellem Forschungsstand nicht. In Schussenried ergab sich dies allein aus dem Umstand, dass der letzte «T4»-Transport nach Grafeneck am 1. November 1940, also an dem Tag, an dem die Südtiroler erst in Schussenried eintrafen, erfolgte. ${ }^{113}$ Aber auch die in Zwiefalten untergebrachten Südtiroler, die in der Zeit, als Zwiefalten hunderte Patienten nach Grafeneck verlegte, dort eintrafen, wurden nicht, wie man vermuten könnte, in die «Aktion T4» einbezogen. Vielmehr wurden drei Südtiroler Psychiatriepatienten aus Zwiefalten, die im Oktober 1940 in einem Sammeltransport in die Tötungsanstalt Grafeneck «verlegt» worden waren, noch am gleichen Tag ohne nähere Erklärung wieder zurückverlegt. ${ }^{114}$

108 Vgl. Schussenried an Württembergisches Innenministerium betr. Überstellung der geisteskranken Umsiedler, 12.2.1942, HStA Stuttgart, E 151/53, Bü 423, Bl. 128f. sowie diesbezüglichen Schriftverkehr der DUS, TLA, Reichsstatthalter, DUS, 58, unpag.

109 DUS an Hall betr. Südtiroler Umsiedler, 3.12.1941, TLA, Reichsstatthalter, DUS, 58, unpag.

110 Vgl. Namensverzeichnisse der am 10. und 11.3.1942 nach Schussenried verlegten Südtiroler, TLA, Reichsstatthalter, DUS, 58 sowie Aufnahmebuch Hall. In den Aufnahmebüchern Schussenried wurden die Transporte unter dem 11.und 12.3.1942 geführt, vgl. Aufnahmebücher Männer (1918-49) und Frauen (1927-45) Schussenried,ZfP Bad Schussenried sowie Heilanstalt Schussenried an Württembergisches Ministerium des Innern betr. Überstellung der geisteskranken Umsiedler, 13.3.1942, HStA Stuttgart, E 151/53, Bü 423, Bl. 132.

111 Stähle an RÄK, 17.8.1942, HStA Stuttgart, E 151/53, Bü 423, B1. 142.

112 Vgl. May 1996b.

113 Vgl. wiederum May1996b.

114 Vgl. Hauptbucheintragungen 3942, 3954 und 3983. Hauptbuch der Heilanstalt Zwiefalten, Männer-Abteilung, 1940-1948, ZfP Südwürttemberg, Standort Münsterklinik Zwiefalten. 
Auch wenn die Südtiroler nach bisherigem Kenntnisstand somit von der «Aktion T4» ausgenommen waren, ist über den gesamten Kriegszeitraum dennoch eine sehr hohe Sterblichkeit unter diesen zu verzeichnen gewesen. So verstarb bis zum Mai 1945 etwa die Hälfte der 299 aus Pergine nach Zwiefalten verlegten Patienten. Diese hohe Sterblichkeit war kein Einzelphänomen der Südtiroler, sie betraf vielmehr alle Patienten gleichermaßen, wobei sie unseren bisherigen Recherchen nach in Zwiefalten deutlich höher lag, als in den vergleichbaren württembergischen Anstalten Schussenried und Weissenau bei Ravensburg. Neben der unzureichenden Versorgung, der schlechten Unterbringungssituation sowie der Tuberkulose war sie hier auch auf die gezielte Überdosierung von Beruhigungsmitteln zurückzuführen, die möglicherweise auch die Südtiroler betraf. ${ }^{115}$ Die Akteneinträge lassen jedoch eine Unterscheidung realer von fingierten Todesursachen nur schwer zu, so dass sich eine Einbeziehung der Südtiroler in die dezentrale bzw. «Medikamenteneuthanasie» zwar vermuten, nicht aber belegen lässt.

Auch im benachbarten Schussenried war die Sterblichkeit unter den Südtirolern hoch. Allerdings scheint dies Folge des Hungers, der Kälte, des Mangels an Pflege, der Überfüllung der Anstalt und der häufigen Tuberkuloseerkrankungen gewesen zu sein und nicht medikamentöser Tötungen. Bis zum Mai 1945 verstarben 41 der 112 im November 1940 nach Schussenried verlegten Südtiroler, und von den im März 1942 aus Hall überstellten 68 Menschen erlebten 17 das Kriegsende nicht. ${ }^{116}$ Eine von ihnen war die Patientin Emilia V., deren Schicksal hier beispielhaft herangezogen sei.

Die Patientenakte mit dem Vermerk «Südtiroler Umsiedlerin» fand sich im Aktenbestand der ehemaligen «Württembergischen Heilanstalt Schussenried». ${ }^{117}$

Die Patientin gehörte zu jenen Südtirolern, die am 1. November 1940 aus Hall nach Schussenried verlegt worden waren. Wenige Monate nach ihrem Eintreffen in Schussenried wurde für sie am 21. Februar 1941 ein Meldebogen der «T4» ausgefüllt, der noch in der Patientenakte vorhanden ist. Darin ist eine Taubstummheit vermerkt, ebenso findet sich darin die Diagnose Schizophrenie. ${ }^{118}$ Der Beruf der Patientin ist mit «Hausgehilfin» angegeben.

115 Vgl.zu Zwiefalten Rüdenburg 1996, hier 44-46. Die medikamentösen Tötungen waren auch Gegenstand des Grafeneck-Prozesses von 1949. Vgl. dazu u.a. Kinzig/Stöckle 2011.

116 Vgl. Aufnahmebücher Männer (1918-1949) und Frauen (1927-1945) Schussenried, Archiv ZfP Südwürttemberg, Standort Bad Schussenried.

117 Diese Akte wurde im heutigen Staatsarchiv (StA) Sigmaringen archiviert (Akten-Nr. Wü 68/1 Acc. 4/1992 3832/5831). Die Akte trägt den gestempelten Vermerk «Südtiroler Umsiedlerin».

118 Meldebogen der Patientin Emilia V. vom 21.2.1941, StA Sigmaringen, Wü 68/1 Acc. 4/1992, 3832/5831. Diese Angaben werden von den Angehörigen bestätigt. Wir danken Frau Carla Giacomozzi, Bolzano/Bozen, für die Übermittlung dieser Informationen. 
Ganz offensichtlich war die Südtirolerin also in den Erfassungsradius der «T4» geraten - in ihren Aktionsradius, das heißt eine ihrer Tötungsanstalten, sollte sie jedoch, wie auch die übrigen Südtiroler, nicht gelangen. Die Tötungsanstalt Grafeneck war zu diesem Zeitpunkt bereits geschlossen worden. Verlegungen in eine andere Tötungsanstalt, z.B. Hadamar, erfolgten nach derzeitigem Forschungsstand nicht. Nichtsdestotrotz hätte sie im Falle einer Wiederaufnahme der Morde sofort verlegt werden können.

Emilia V. verblieb also in Schussenried. Ihre Angehörigen erkundigten sich in regelmäßigen Abständen nach ihrem Befinden und erhielten von der behandelnden Ärztin entsprechende Antwortschreiben. In einem dieser Schreiben berichtete diese u.a., dass die Patientin kein Deutsch verstünde, was einerseits die Behandlung und das Leben auf der Station schwierig gestalten würde, und in zweiter Hinsicht eine «Option» für das Deutsche Reich wenig plausibel erscheinen lässt. ${ }^{119}$ Was die Frage der Einbürgerung anbelangt, so dürfte sich vor allem der Umstand, dass man sie 1941 aus ärztlicher Sicht für «unzurechnungsfähig» erklärt hatte, negativ ausgewirkt haben. Andererseits ist in der gleichen Korrespondenz zwischen dem zuständigen Gauleiter und der DUS vom 20. Juni 1941 vermerkt: «Es besteht jedoch kein Anlass, [die Patientin] nicht so wie alle übrigen Südtiroler zu behandeln.» ${ }^{120}$ Die Einbürgerung erfolgte allerdings nicht. Zum medizinischen Verlauf der sog. Therapie gibt die Akte folgende Auskunft: die Patientin sei an einem «akuten Lungenprozess» erkrankt. Bei «ihrem stark reduzierten Kräfte- und Ernährungszustand [sei], wenn nicht bald eine Änderung eintritt, mit einem raschen Ableben zu rechnen». ${ }^{121}$ Weitere Angaben zu medizinischer Behandlung und Verlauf der Erkrankung sind in dieser Akte spärlich bis nicht vorhanden. In einem Schreiben vom 29. Juni 1943 an die DUS wird dann neben der Todesnachricht mit angegebenem Datum auch von einer Beerdigung in Schussenried berichtet, die für den 2. Juli 1943, 13.00 Uhr, vorgesehen gewesen sein soll. Der Vater der Patientin sei von diesem Termin der Beerdigung in Kenntnis gesetzt worden. ${ }^{122}$ Angesichts des ärztlichen Aktenvermerks, der sich in dieser Form beinah standardisiert auch in zahlreichen anderen Akten finden lässt, und der Todesursache, drängt sich der Verdacht auf, dass der Tod der Patientin herbeigeführt worden

119 Krankenakte der Patientin Emilia V., StA Sigmaringen, Wü 68/1 Acc. 4/1992, 3832/5831.

120 Anstalt Schussenried an die DUS, 20.6.1941, sowie StA Sigmaringen, Wü 68/1 Acc. 4/1992, 3832/5831. In einem Briefwechsel vom Juli 1943, bereits nach dem Tod der Patientin, ist darüber hinaus von einem Paket die Rede, dass ein Angehöriger vermisse.

121 Wiederum in der Krankenakte von Emilia V., StA Sigmaringen, Wü 68/1 Acc. 4/1992, 3832/5831, unter «Therapie».

122 Schussenried an die DUS, 29.6.1943, StA Sigmaringen, Wü 68/1 Acc. 4/1992, 3832/5831. 
sein könnte. Konkrete Belege, dafür, dass es sich nicht um eine «normale» Lungenerkrankung gehandelt hat, liegen jedoch nicht vor. Zudem sind derartige Tötungen in Schussenried bislang nicht bekannt geworden, auch wenn sie deshalb nicht auszuschließen sind.

\section{«Rückführungen» und Repatriierungsversuche vor und nach 1945}

Bereits im Januar 1941 wurden vier Südtiroler Psychiatriepatienten aus Zwiefalten und einer aus Schussenried auf Anordnung der DUS nach Pergine zurückverlegt, da die Angehörigen nicht optiert hatten. Eine Rückführung nach Italien sollte jedoch die Ausnahme bleiben, ebenso wie Entlassungen. Lediglich etwa 30 Südtiroler Psychiatriepatienten aus Schussenried und 8 aus Zwiefalten wurden vor 1945 zu ihren Angehörigen - Optanten, die im Deutschen Reich angesiedelt worden waren «nach Hause» entlassen. ${ }^{123}$ Nach dem Kriegsende wurden in Schussenried weitere 8 Südtiroler Psychiatriepatienten auf Drängen ihrer Angehörigen entlassen (fünf nach Italien, zwei nach Österreich und einer nach Deutschland). ${ }^{124}$

Nach Kriegsende bestand formal die Möglichkeit zur «Rückoption», die den Patienten allerdings verwehrt blieb, da sie zum überwiegenden Teil nicht die deutsche Staatsbürgerschaft erworben hatten und damit auch nicht «rückoptieren» konnten. ${ }^{125}$ Die Nachkriegsjahre mit dem Besatzungsstatus brachten schließlich eine weitgehende Unterbrechung der Briefkontakte und Besuche der Angehörigen. Mit dem Bekanntwerden der nationalsozialistischen Krankenmorde glaubten offenbar auch viele Südtiroler Familien, ihre Angehörigen seien ermordet worden. Ab Mitte der 1970er Jahre, mit der Lockerung der Grenzen und den Fortschritten bei den Nachrichten- und Verkehrsverbindungen, wurde in Schussenried begonnen, die noch lebenden Südtiroler Psychiatriepatienten auch für längere Zeit bei aufnahmebereiten Angehörigen zu beurlauben. Federführend waren bei diesen Aktionen der dortige Oberpfleger Albert Altherr und der Psychiater Dr. Johannes May, die ab 1974 auch jährliche Urlaubsfahrten für Patientinnen und Patienten nach Südtirol organisierten.Zu diesem Zeitpunkt lebten in Schussenried und Zwiefalten von den 494 Südtiroler Psychiatriepatientinnen und -patienten

123 Vgl. May 1995.

124 Vgl. handschriftliche Aufzeichnungen von Johannes May, Ordner Südtiroler Schussenried (nicht paginiert), Nachlass Johannes May im Archiv des ZfP Südwürttemberg, Standort Münsterklinik Zwiefalten.

125 Vgl. weiter May 1995. 
nur noch 37 Menschen. ${ }^{126}$ Nur bei 7 von ihnen waren die Angehörigen bekannt, über diese Angehörigen konnten aber schließlich weitere 26 Familien ermittelt werden. Insgesamt wurden bis 1988 noch insgesamt 15 einwöchige Urlaubsfahrten durchgeführt, bei denen es den Zeitzeugen zufolge zu berührenden Begegnungen nach über 30 Jahren Abwesenheit gekommen sein soll. ${ }^{127}$

Das Ergebnis dieser Repatriierungsversuche war, dass ab 1982 acht Südtiroler Psychiatriepatienten zurück vermittelt werden konnten und bei zwei weiteren Patienten die Aufenthaltsgenehmigung für einen Urlaub in eine solche des Daueraufenthalts umgewandelt wurde. ${ }^{128}$

\section{Fazit}

Die Umsiedlung psychisch kranker und geistig behinderter Südtiroler, die seitens der deutschen und der italienischen Umsiedlungsakteure als bequeme «Verschiebemasse» zur Erfüllung der Abwanderungsquoten betrachtet wurden, zeigt deutlich, mit welcher Totalität erfasst und «ausgesondert» wurde. «Ausgesondert» wurden dabei alle diejenigen, die als «unerwünschter Bevölkerungszuwachs» galten. Sie sollten nicht nur nicht eingebürgert, sondern oftmals auch dauerhaft psychiatrisiert werden. Der Optionscharakter der Umsiedlung war dabei letztlich nur Staffage: nichtoptionsberechtigte Anstaltspatienten wurden aus umsiedlungspolitischem Kalkül heraus dennoch umgesiedelt, optionsberechtigte, aber «unerwünschte» Südtiroler zwar ins Deutsche Reich umgesiedelt, dort jedoch nicht eingebürgert.

Für alle in die psychiatrischen Anstalten des Deutschen Reiches «umgesiedelten» Südtiroler bedeutete die Verlegung angesichts der NS-Kranken-

126 Die Mehrzahl der Patienten war zu diesem Zeitpunkt bereits verstorben. 39 Südtiroler wurden aus Schussenried seit 1945 entlassen oder in andere Einrichtungen verlegt, aus Zwiefalten 30. Ihr weiteres Schicksal ist unbekannt. Vgl. Aufnahmebücher Männer (1918-1949) und Frauen (1927-1945) Schussenried, Archiv ZfP Südwürttemberg, Standort Bad Schussenried sowie Hauptbuch der Heilanstalt Zwiefalten, Männer-Abteilung, 1940-1948, sowie Hauptbuch der Frauen-Abteilung 1939-1949, ZfP Südwürttemberg, Standort Münsterklinik Zwiefalten.

127 Einige der Reiseberichte wurden in der Hauszeitschrift des seinerzeitigen PLK Bad Schussenried Schussenrieder Blätter veröffentlicht, und sind im Archiv des ZfP Südwürttemberg einsehbar. Die fast vollständige Sammlung befindet sich im Nachlass von Johannes May.

128 Sechs Südtiroler Psychiatriepatienten erhielten Heimplätze in der Nähe der Angehörigen und zwei wurden in ihren Familien aufgenommen. Eigene Erhebungen und handschriftliche Aufzeichnungen von Johannes May, Ordner Südtiroler Fahrten (nicht paginiert). Vgl. Nachlass Johannes May im Archiv des ZfP Südwürttemberg, Standort Münsterklinik Zwiefalten. 
morde eine existentielle Bedrohung. Dass der überwiegende Teil der aus Südtirol stammenden Patientinnen und Patienten schließlich letztlich weder in Hall noch in den württembergischen Heilanstalten Opfer der «Aktion T4» wurde, war zum Teil möglicherweise außenpolitischem Kalkül geschuldet. Von der «T4» erfasst wurden die Südtiroler allerdings sehr wohl, so dass sie bei einer möglichen Wiederaufnahme der zentralen Morde, wie sie von der «T4» beabsichtigt wurde, sofort hätten einbezogen werden können.

Auch wenn die Südtiroler im Vergleich zu anderen Patienten und bezogen auf die «Aktion T4» also eine Sonderrolle einnahmen, so teilten sie doch zumindest in Bezug auf die sich zunehmend verschlechternde Versorgungsund Unterbringungssituation das Schicksal aller übrigen Patienten, die der «Aktion T4» entgangen waren, und denen für weitere Jahre die Gefahren von medizinischer Vernachlässigung, «forciertem Hungersterben» oder gar aktiverer Tötungsmaßnahmen der dezentralen «Euthanasie» drohten. ${ }^{129}$ Das Schicksal derjenigen Optanten aus Südtirol, die im Zuge des Optionsabkommens in Anstalten nach Württemberg verlegt wurden, war Gegenstand der vorliegenden Studie. Das Schicksal derjenigen Südtiroler, die Südtirol in diesem Sinne zwar verließen, jedoch in die Universitätsklinik nach Innsbruck und in die Heilanstalt Hall verlegt wurden und dort verblieben, ist bisher nicht untersucht worden und stellt unserer Ansicht nach ein forschungswürdiges Desiderat dar.

\section{Bibliographie}

Primärliteratur:

Handausgabe der Umsiedlungs-Bestimmungen für die deutschen Optanten, hrsg. vom Leiter der Amtlichen Deutschen Ein- und Rückwandererstelle (Bozen 1940)

Reichskommissar für die Festigung Deutschen Volkstums (Hrsg.), Der Menscheneinsatz. Grundsätze, Anordnungen und Richtlinien (Berlin 1940)

Sekundärliteratur:

Alexander, Helmut, «Die Umsiedlung der Südtiroler 1939-1945», in: Helmut Alexander/Stefan Lechner/Adolf Leidlmair, Heimatlos. Die Umsiedlung der Südtiroler (Wien 1993) 43-179

Aly, Götz, «Endlösung». Völkerverschiebung und der Mord an den europäischen Juden (Frankfurt/M. 1998)

Arreghini, Ermanno, «Option: eine freie Entscheidung oder Abschiebung von «Unzurechnungsfähigen ??», in: Verband Angehöriger und Freunde psychisch Kranker (Hrsg.), Wahnsinn

129 «Euthanasie»-Morde an Südtiroler Patientinnen und Patienten lassen sich im Einzelfall und zum gegenwärtigen Zeitpunkt nicht belegen. Allerdings deuten manche Praktiken darauf hin. Auch die von der Forschung herausgearbeitete Rolle Zwiefaltens innerhalb der dezentralen Morde lässt eine Einbeziehung der Südtiroler in diese Krankenmordaktion möglich erscheinen: Zwiefalten gilt als Ort der «Medikamenteneuthanasie». Für diesbezügliche Diskussion danken wir Georg Lilienthal, Gedenkstätte Hadamar sowie Thomas Stöckle, Gedenkstätte Grafeneck. 
und ethnische Säuberung. Deportation und Vernichtung psychisch Kranker aus Südtirol 1939-45 (Bozen 1995) 37-41

Demetz, Josef, «Erinnerungen von Josef Demetz, Südtiroler Patient der Anstalt St. Pietro in Pergine», in: Hermann J. Pretsch (Hrsg.), Euthanasie. Krankenmorde in Südwestdeutschland (Zwiefalten 1996) 110-115

Dietrich-Daum, Elisabeth u.a. (Hrsg.), Psychiatrische Landschaften. Die Psychiatrie und ihre Patientinnen und Patienten im historischen Raum Tirol seit 1830 (Innsbruck 2011)

Faulstich, Heinz, Hungersterben in der Psychiatrie 1914-1949. Mit einer Topographie der NSPsychiatrie (Freiburg i.Br. 1998)

Fiebrandt, Maria, «Volks- und Reichsdeutsche in den Heilanstalten Warta und Tiegenhof (Warthegau) 1939-1945», in: Arbeitskreis zur Erforschung der nationalsozialistischen «Euthanasie» und Zwangssterilisation (Hrsg.), NS-Euthanasie in der «Ostmark» (Münster 2012) 219-238

Fuchs, Petra u.a. (Hrsg.), «Das Vergessen der Vernichtung ist Teil der Vernichtung selbst». Lebensgeschichten von Opfern der nationalsozialistischen «Euthanasie» (Göttingen 2007)

Gutberger, Hansjörg, «Auf dem Weg zu einer radikalen Ordnung des Sozialen: Nationalsozialistische Raumforschung, Raumordnung und ländliche Sozialwissenschaft vor Beginn der NS-Siedlungspolitik im Zweiten Weltkrieg», Geschichte und Region/Storia e regione 18, Heft 2 (2009) 21-47

Hecker, Hellmuth, Die Umsiedlungsverträge des Deutschen Reiches während des Zweiten Weltkrieges (Hamburg 1971)

Heinemann, Isabel/Patrick Wagner (Hrsg.), Wissenschaft. Planung. Vertreibung. Neuordnungskonzeptionen und Umsiedlungspolitik im 20. Jahrhundert, Beiträge zur Geschichte der Deutschen Forschungsgemeinschaft 1 (Stuttgart 2006)

Hinterhuber, Hartmann, Ermordet und vergessen. Nationalsozialistische Verbrechen an psychisch Kranken und Behinderten in Nord- und Südtirol (Innsbruck 1995)

Jütte, Robert (Hrsg.), Medizin und Nationalsozialismus. Bilanz und Perspektiven der Forschung (Göttingen 2011)

Karlegger, Selma, Südtiroler Kinder und Jugendliche als Opfer der «NS-Euthanasie», Diplomarbeit (Innsbruck 2006)

Kepplinger, Brigitte, «NS-Euthanasie in Österreich. Die «Aktion T4» - Struktur und Ablauf», in: Brigitte Kepplinger/Gerhart Marckhgott/Hartmut Reese (Hrsg.), Tötungsanstalt Hartheim, (Linz $\left.{ }^{2} 2008\right)$ 35-62

Kinzig, Jörg/Thomas Stöckle (Hrsg.), 60 Jahre Tübinger Grafeneck-Prozess. Betrachtungen aus historischer, juristischer, medizinethischer und publizistischer Perspektive. (Zwiefalten 2011)

Kotzian, Ortfried, Die Umsiedler. Die Deutschen aus West-Wolhynien, Galizien, der Bukowina, Bessarabien, der Dobrudscha und in der Karpatenukraine, Studienbuchreihe der Stiftung Ostdeutscher Kulturrat 11 (München 2005)

Kretschmer, Manfred, «Von der Staatsirrenanstalt zum Akademischen Krankenhaus, III. Die Heilanstalt im Hitler-Reich», in: Peter Eitel (Hrsg.), Weissenau. Geschichte und Gegenwart (Sigmaringen 1983) 337-354

Latour, Conrad F., Südtirol und die Achse Berlin-Rom 1938-1945 (Stuttgart 1962)

Leidlmair, Adolf, «Die Durchführung der Option und ihr Ergebnis», in: Rudolf Lill, Die Option der Südtiroler 1939 (Bozen 1991) 177-199

Leniger, Markus, Nationalsozialistische «Volkstumsarbeit» und Umsiedlungspolitik 1933-1945 (Berlin 2006)

Ley, Astrid, «Der Beginn der NS-Krankenmorde in Bandenburg an der Havel. Zur Bedeutung der «Brandenburger Probetötung» für die «Aktion T4»», Zeitschrift für Geschichtswissenschaft 58 Heft 4 (2010) 321-331

Longerich, Peter, Politik der Vernichtung. Eine Gesamtdarstellung der nationalsozialistischen Judenverfolgung (München/Zürich 1998)

May, Johannes, «Südtiroler Kranke in Zwiefalten und Schussenried», in:Hermann J.Pretsch (Hrsg.), «Euthanasie». Krankenmorde in Südwestdeutschland (Zwiefalten 1996) 69-74 (May 1996a)

May, Johannes, «Die Staatliche Heilanstalt Schussenried in den Jahren 1933 bis 1945», in: Hermann J. Pretsch (Hrsg.), «Euthanasie». Krankenmorde in Südwestdeutschland (Zwiefalten 1996) 74-83 (May 1996b) 
May, Johannes, «Südtiroler Patienten in Baden-Württemberg. Versuche ihrer Repatriierung», in: Verband Angehöriger und Freunde psychisch Kranker in Südtirol (Hrsg.), Wahnsinn und ethnische Säuberung. Deportation und Vernichtung psychisch Kranker aus Südtirol 1939-1945 (Bozen 1995) 55-64

Müller, Christine-Ruth/Hans-Ludwig Siemen, Warum sie sterben mußten. Leidensweg und Vernichtung von Behinderten aus den Neuendettelsauer Pflegeanstalten im «Dritten Reich» (Neustadt a. d. Aisch 1991)

Müller, Roland (Hrsg.), Krankenmord im Nationalsozialismus. Grafeneck und die «Euthanasie» in Südwestdeutschland (Stuttgart 2001)

Müller, Thomas/Thomas Beddies, «'Life unworthy of living'. Psychiatry in National Socialist Germany», International Journal for Mental Health vol. 35 no. 3 (2006) 94-104

Müller, Thomas/Thomas Beddies, «Psychiatrie und Psychotherapie im nationalsozialistischen Deutschland.Teil I:Die Psychiatrie,Psychologische Medizin», Österreichische Fachzeitschrift für medizinische Psychologie, Psychosomatik und Psychotherapie 15 (2004) 16-23

Pantozzi, Giuseppe, Die brennende Frage. Geschichte der Psychiatrie in den Gebieten von Bozen und Trient (1830-1942) (Bozen 1989)

Pantozzi, Giuseppe, «La deportazione in Germania die malati di mente durante la Seconda Guerra Mondiale», Studi Trentini di Scienze Storiche, A. LXXV, Sezione 1-4 (Trento 1996) 367-396

Rexer, Martin, «Die 〈Aktion T4〉 in den Zwischenanstalten Zwiefalten und Schussenried», in: Hermann J. Pretsch, (Hrsg.), Euthanasie. Krankenmorde in Südwestdeutschland (Zwiefalten 1996) 27-37

Rieß,Volker, Die Anfänge der Vernichtung «lebensunwerten Lebens» in den Reichsgauen DanzigWestpreußen und Wartheland 1939/40 (Frankfurt/M. 1995)

Rotzoll, Maike u.a. (Hrsg.), Die nationalsozialistische «Aktion T4» und ihre Opfer. Historische Bedingungen und ethische Konsequenzen für die Gegenwart (Paderborn 2010)

Rüdenburg, Bodo, «Die «Aktion T4» in Zwiefalten», in: Hermann J. Pretsch (Hrsg.), Euthanasie. Krankenmorde in Südwestdeutschland (Zwiefalten 1996) 37-46

Seifert, Oliver, «Sterben hätten sie auch hier können.» Die «Euthanasie»-Transporte aus der Heil- und Pflegeanstalt Hall in Tirol nach Hartheim und Niedernhart», in: Brigitte Kepplinger/Gerhart Marckhgott/Hartmut Reese (Hrsg.), Tötungsanstalt Hartheim (Linz ${ }^{2} 2008$ ) 359-410

Steinert, Tilman, Die Geschichte des Psychiatrischen Landeskrankenhauses Weissenau. Darstellung der Anstaltsgeschichte von 1888 bis 1945 im ideengeschichtlichen und sozio-ökonomischen Kontext, Diss. med. (Ulm 1985)

Stuhlpfarrer, Karl, Umsiedlung Südtirol 1939-40 (Wien/München 1985)

Süß, Winfried, Der «Volkskörper» im Krieg. Gesundheitspolitik, medizinische Versorgung und Krankenmord im nationalsozialistischen Deutschland 1939-1945 (München 2003)

Verband Angehöriger und Freunde psychisch Kranker (Hrsg.), Wahnsinn und ethnische Säuberung. Deportation und Vernichtung psychisch Kranker aus Südtirol 1939-45 (Bozen 1995)

Wedekind, Michael, ««Volksgemeinschaft», NS-Raumpolitik und Migrationsverhältnisse: Südtiroler Umsiedlungsplanungen (1939-1945)», in: Jochen Oltmer (Hrsg.), Nationalsozialistisches Migrationsregime und «Volksgemeinschaft» (Paderborn 2012) 265-294

Wedekind, Michael, «Planung und Gewalt: Raumordnung und Bevölkerungsplanung im Kontext der Umsiedlung Tirol», Geschichte und Region/Storia e regione 18, Heft 2 (2009) 71-109

\section{Quellenverzeichnis}

Archivum Państwowe w Poznaniu (APP)

Bayerisches Hauptstaatsarchiv München (BayHStA)

Bundesarchiv Berlin (BA Berlin)

Hauptstaatsarchiv Stuttgart (HStA Stuttgart)

Staatsarchiv Sigmaringen (StA Sigmaringen)

Tiroler Landesarchiv Innsbruck (TLA)

Zentrum für Psychiatrie Südwürttemberg (ZfP) 